\title{
REVIEW ARTICLE OPEN Charge and phonon transport in PbTe-based thermoelectric materials
}

\author{
Yu Xiao ${ }^{1}$ and Li-Dong Zhao ${ }^{1}$
}

$\mathrm{PbTe}$ is a typical intermediate-temperature thermoelectric material, which has undergone extensive developments and achieved excellent high thermoelectric performance. In this perspective we summarized several strategies that were successfully applied in PbTe-based thermoelectric materials through manipulating charge and phonon transports, such as optimizing carrier density to tune Fermi level, tailoring band structure to enhance effective mass, and designing all-scale hierarchical architectures to suppress phonon propagation. Meanwhile, due to the different features of conduction and valence bands, we separately introduced the approaches to enhance performance of p-type and n-type PbTe. In p-type PbTe, the strategies of band convergence, band alignment and density of state (DOS) distortion are more effective to achieve high electrical transport properties. By contrast, flattening conduction bands and introducing deep impurity level are more suitable for n-type PbTe. Lastly, several potential strategies were proposed to further improve the thermoelectric performance of PbTe-based materials, which might be extended to other thermoelectric systems.

npj Quantum Materials (2018)3:55 ; doi:10.1038/s41535-018-0127-y

\section{INTRODUCTION}

With more than $60 \%$ of input energy being lost, most of which as waste heat, there is a compelling need for high performance thermoelectric materials that can directly and reversibly convert heat to electric energy. With continuing vigorous developments over the past decade, encompassing diverse strategies to enhance the power factor and reduce the thermal conductivity, the field of thermoelectrics has truly reached its Renaissance era. However, practical realization of thermoelectric materials is limited by hitherto low dimensionless figure of merit, $Z T$, which is governed by Carnot efficiency of waste heat conversion. ${ }^{1}$ Namely, $Z T=S^{2} \sigma T /$ $\kappa$, where $S, \sigma, T$, and $\kappa$ denote the Seebeck coefficient, electrical conductivity, working temperature in Kelvin, and thermal conductivity, respectively. ${ }^{2}$ Apparently, a good thermoelectric material simultaneously demands large Seebeck coefficient, high electrical conductivity and low thermal conductivity. However, the complex relationship among these thermoelectric parameters makes it difficult to enhance the final $Z T$ value. Therefore, countless efforts are aimed to improve the $Z T$ value through balancing these interdependent thermoelectric parameters.

After predicating and witnessing that narrow-gap semiconductors were excellent thermoelectric materials, the development of thermoelectric materials experienced a slow progress. ${ }^{3,4}$ The idea of "size effect" promotes a rapid progress due to dramatically enhanced thermoelectric performance through reducing lattice thermal conductivity via nanostructuring. ${ }^{5,6}$ Before the "size effect" was employed in bulk materials, most reports focused on its quantum effects in low-dimensional materials such as superlattice thin films and nanowires. ${ }^{7-9}$ When the "size effect" approaches atomic-scale, quantum confinements will produce remarkable effects on charge and phonon transports in thermoelectric materials. ${ }^{10,11}$ The idea of "size effect" substantially motivated researchers to optimize thermoelectric performance, such as hierarchical architecturing and band structure engineering. Based on above strategies, many promising thermoelectric materials emerged in an endless stream nowadays. Specifically, near room temperature (300-500 K) thermoelectrics include $(\mathrm{Bi}, \mathrm{Sb})_{2}(\mathrm{Se}, \mathrm{Te})_{3^{-}}$ based alloys and MgAgSb alloys. ${ }^{12-15}$ Intermediate-temperature (500-900 K) thermoelectrics include $\mathrm{Pb}(\mathrm{Te}, \mathrm{Se}, \mathrm{S}),{ }^{16-20} \mathrm{Sn}(\mathrm{Se}, \mathrm{S}){ }^{21-}$ $27 \mathrm{SnTe}_{1}{ }^{28,29} \mathrm{GeTe}^{30} \mathrm{Cu}_{2}(\mathrm{Te}, \mathrm{Se}, \mathrm{S}){ }^{31,32} \mathrm{Mg}_{2}(\mathrm{Si}, \mathrm{Sn}, \mathrm{Ge}){ }^{33,34}$ BiCuSeO, ${ }^{35,36} \mathrm{Zn}_{4} \mathrm{Sb}_{3}{ }^{37} \mathrm{In}_{4} \mathrm{Se}_{3},{ }^{38} \mathrm{Ba}_{8} \mathrm{Ga}_{16} \mathrm{Ge}_{30},{ }^{39}$ skutterudites, ${ }^{40-}$ ${ }^{42}$ and tetrahedrites. ${ }^{43}$ At high temperature $(>900 \mathrm{~K}), \mathrm{SiGe}^{44}(\mathrm{Pr}$, $\mathrm{La}_{3} \mathrm{Te}_{4}{ }^{45,46} \mathrm{Yb}_{14} \mathrm{MnSb}_{11}{ }^{47}$ and half-Heusler (HH) alloys ${ }^{48-50}$ are very promising thermoelectric candidates.

Among well-known thermoelectric materials, $\mathrm{PbTe}$ is recognized as a very promising compound for power generation in intermediate-temperature range. PbTe-based thermoelectric materials have successfully served in several NASA space missions. ${ }^{17}$ In 1960s, due to the technological limits, the thermal conductivity was overestimated, thus the maximum $Z T$ ( $Z T_{\max }$ ) values $\sim 0.7-0.8$ in PbTe were underestimated. After laser flash method was provided to determine thermal diffusivity, ${ }^{51}$ the $Z T_{\max }$ values in both $\mathrm{n}$ - and $\mathrm{p}$-type $\mathrm{PbTe}$ were recognized to be as high as $\sim 1.4 .^{52}$ Generally, the thermoelectric performance in PbTebased materials experienced a large progress especially in recent decades, as shown in Fig. 1. The $Z T_{\max }$ values of p-type PbTe are boosted from $\sim 1.0$ to $\sim 2.5,{ }^{53}$ and the $Z T_{\text {max }}$ values in n-type PbTe reach $\sim 1.8-2.2 .^{16,54,55}$ Notably, it is necessary to maximize the efficiency for a thermoelectric device through developing the well-matched performance for both $\mathrm{p}$ - and n-type PbTe.

Although $\mathrm{PbTe}$ is a simple binary compound, its performance stands out among intermediate-temperature thermoelectrics. $\mathrm{PbTe}$ possesses several outstanding features that are typical requirements for thermoelectrics, such as high Seebeck coefficient due to its complicated electronic band structures, superior electrical conductivity from high-symmetry cubic crystal structure,

\footnotetext{
${ }^{1}$ School of Materials Science and Engineering, Beihang University, Beijing 100191, China
}

Correspondence: L-D. Zhao (zhaolidong@buaa.edu.cn)

Received: 29 May 2018 Revised: 19 September 2018 Accepted: 11 October 2018

Published online: 01 November 2018 
and low thermal conductivity caused by strong anharmonicity due to the $\mathrm{Pb}$ local off-center. ${ }^{56-58}$ To date, PbTe is still a research hot spot in thermoelectric community. After a good perspective summary in $2011,{ }^{17}$ a large amount of achievements have been reported in PbTe-based thermoelectrics, which are evidenced by $Z T_{\max } \sim 2.2$ in p-type PbTe-SrTe in $2012,{ }^{59} Z T_{\max } \sim 2.0$ in p-type PbTe-MgTe in $2013,{ }^{60} Z T_{\max }>2.0$ in p-type PbTe-PbS in $2014,{ }^{61}$ $Z T_{\max } \sim 2.5$ in p-type PbTe-SrTe in $2016,{ }^{53} Z T_{\max } \sim 1.8$ in n-type PbTe-InSb, ${ }^{54}$ and $Z T_{\max } \sim 1.8$ in n-type PbTe-Sb-I in $2017,{ }^{55}$ et al. Therefore, it is essential to update these research progresses in PbTe-based thermoelectrics.

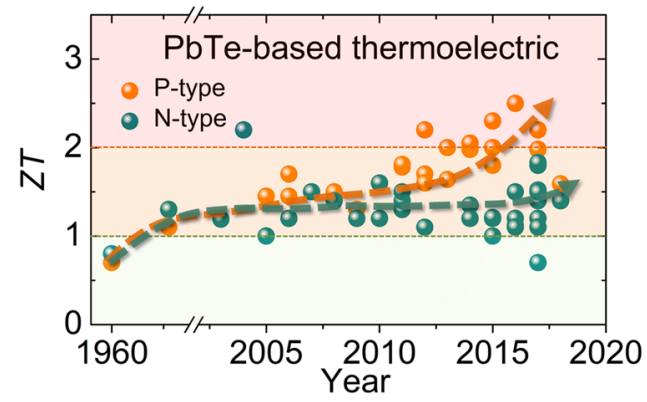

Fig. 1 Timeline of $Z T$ values achieved in representative PbTe-based thermoelectrics, including both p-type (orange ball) and n-type PbTe (green ball)

(a)

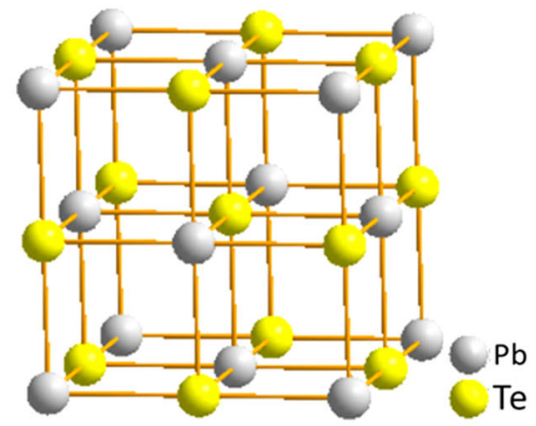

(c)

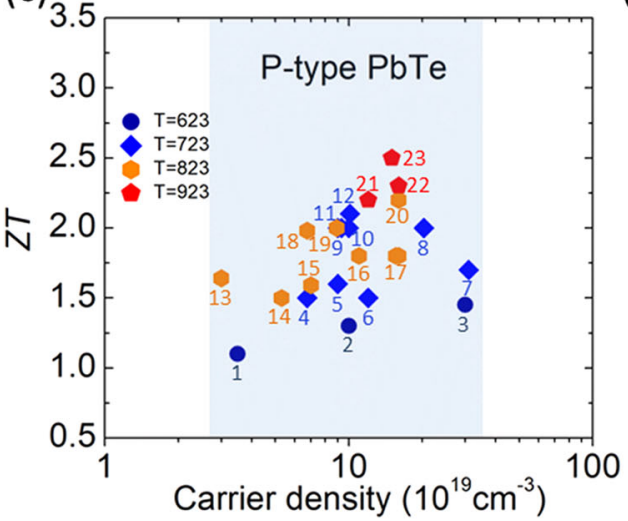

In this perspective, we summarized the effective strategies to improve thermoelectric performance of PbTe-based systems, including optimizing carrier density to tune Fermi level, tailoring band structures to enhance effective mass, and designing all-scale hierarchical architectures to suppress phonon propagation. Considering the difference between valence and conduction bands in $\mathrm{PbTe}$, the special emphasis is given on the different strategies to enhance effective mass and Seebeck coefficients by manipulating the band structures in p-type and n-type PbTe, respectively. In p-type PbTe, the convergence in light $(L)$ and heavy $(\Sigma)$ valence bands and DOS distortion in valence band are extensively utilized. ${ }^{61-64}$ Additionally, band alignment also plays a positive role in maintaining carrier mobility when one second phase is embedded in p-type PbTe. ${ }^{53,65}$ On the contrary, because of the large offset between light $(L)$ and heavy $(\Sigma)$ conduction bands, it is challenging to achieve conduction bands convergence in n-type PbTe. However, the approaches of conduction band flattening ${ }^{66}$ and introducing deep impurity level ${ }^{67,68}$ could boost power factor in n-type PbTe. Apart from the discussions about electrical transport properties, we also put emphasis on suppressing phonon propagation through designing hierarchical architectures ranging from atomic-scale, nanoscale to mesoscale. We believe that this perspective could provide useful insights into PbTe-based systems, which can be extended to other thermoelectric systems. At last, a discussion of future possible strategies is proposed to aim at further enhancing the thermoelectric performance in $\mathrm{PbTe}$-based materials.

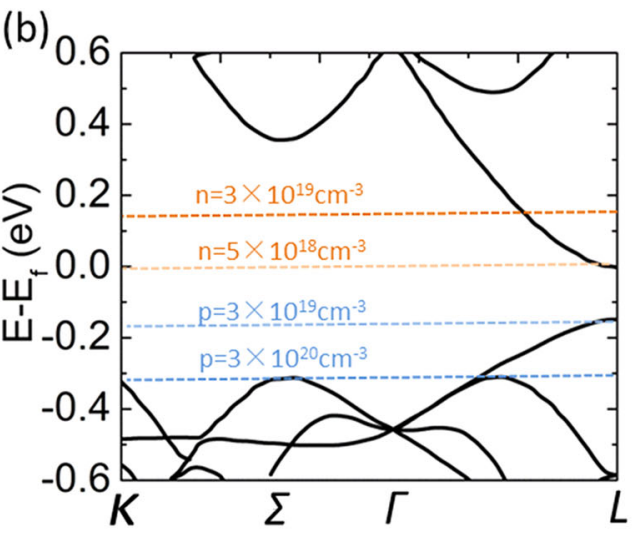

(d)

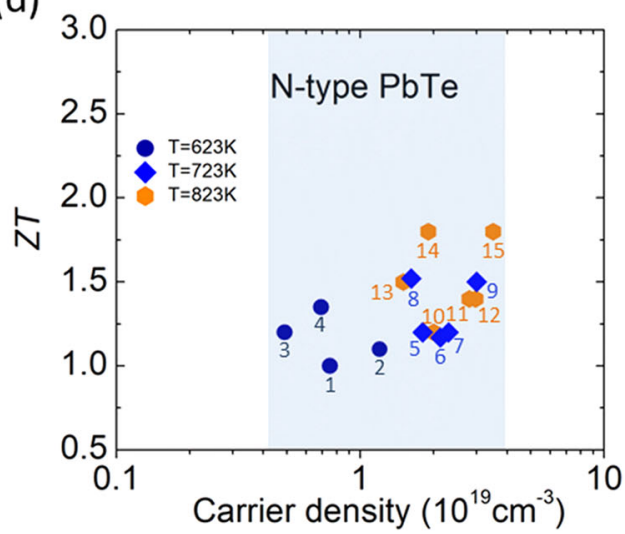

Fig. 2 a Crystal structure; b electronic band structure; c peak $Z T$ values as a function of optimized carrier density in p-type PbTe systems: (1) PbTe-Ge:Na, ${ }^{101}$ (2) PbTe:(Na, Bi), ${ }_{102}^{102}$ (3) PbTe-Sn:(Ag, Sb), ${ }^{103}$ (4) PbTe-Mn:Na, ${ }^{104}$ (5) PbTe-Se:K, ${ }^{105}$ (6) PbTe-Ca:Na, ${ }_{10}$ (7) PbTe-Cd:Na, ${ }^{92}$ (8) PbTe-S:K, ${ }^{61}$ (9) PbTe:Na, ${ }^{106}$ (10) PbTe:Na (Nano-precipitates), ${ }^{107}$ (11) PbTe-Ge, ${ }_{112}^{108}$ (12) PbTe-Se-S:Na, ${ }_{109}{ }^{109}$ (13) PbTe-Hg: Na, ${ }_{1}^{110}$ (14) PbTe:Tl, ${ }^{64}$ (15) PbTe-Sn-Se-S: $\mathrm{Na}{ }^{95}$ (16) PbTe-Yb:Na, ${ }^{111}$ (17) PbTe-Se:Na, ${ }^{62}$ (18) PbTe-Mn-Sr:Na, ${ }^{112}$ (19) PbTe-Mg:Na, ${ }^{60}$ (20) PbTe-Eu:Na, ${ }^{113}$ (21) PbTe-Sr:Na, ${ }^{59}$ (22) PbTe-S:Na, ${ }^{63}$ (23) PbTe-Sr:Na (Non-equilibrium) ${ }^{53}$ d peak $Z T$ values as a function of optimized carrier density in n-type PbTe systems: (1) PbTe-Se:Cr ${ }^{114}$ (2) PbTe-Se-S:I, ${ }^{115}$ (3) PbTe-NaCl, ${ }^{116}$ (4) PbTe-Zn:I, ${ }^{117}$ (5) PbTe-Cd:I, ${ }^{118}$ (6) PbTe-Sn-Se:I, ${ }^{119}$ (7) PbTe-Mg:l, ${ }^{120}$ (8) PbTe-Se-S:Cl, ${ }^{121}$ (9) PbTe:Cd, ${ }^{122}$ (10) PbTe:La, ${ }^{123}$ (11) PbTe:(I, In), ${ }^{67}$ (12) PbTe-S:Sb, ${ }^{66}$ (13) PbTe-Cu:I, ${ }^{98}$ (14) PbTe:(Sb, I), ${ }^{55}$ (15) PbTe:(Sb, In). ${ }^{54}$ That shows the large difference of optimized carrier density between p-type $\left(3 \sim 40 \times 10^{19} \mathrm{~cm}^{-3}\right)$ and n-type $\left(0.4 \sim 4 \times 10^{19} \mathrm{~cm}^{-3}\right)$ 


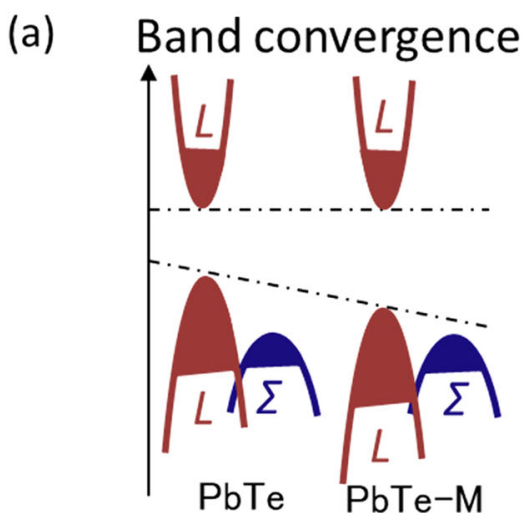

(c)

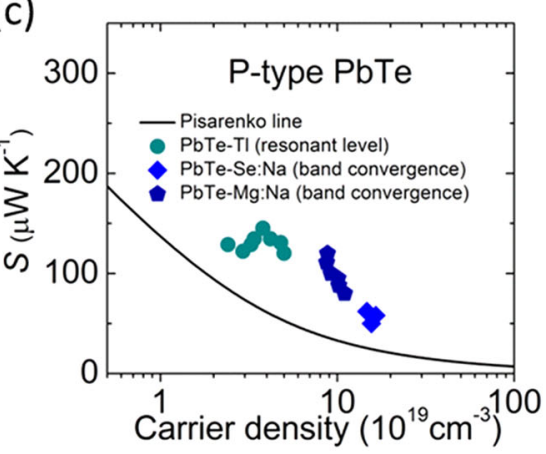

(e)

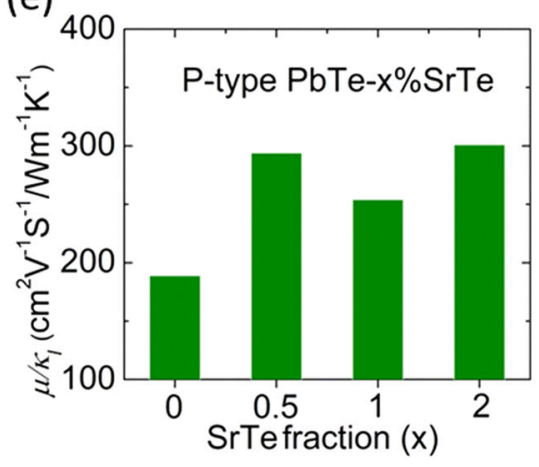

(b)

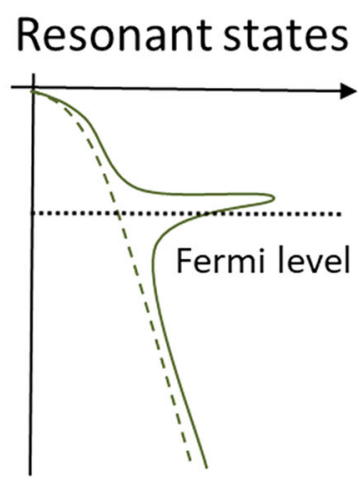

(d)
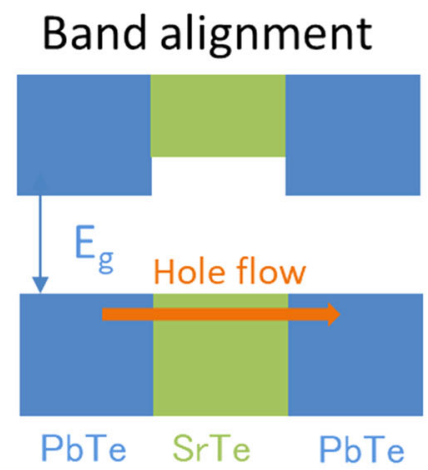

(f)

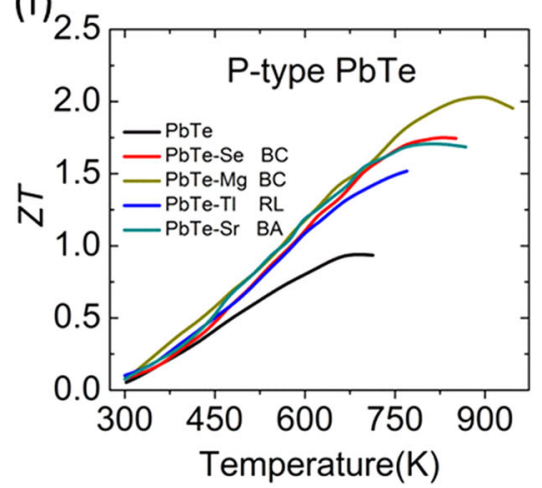

Fig. 3 a Schematic image of band convergence; b Schematic image of DOS distortion in PbTe; c theoretical Pisarenko line shows single parabolic band with effective mass $m^{*}=0.3 m_{e}$ at $300 \mathrm{~K}$, and the point data include PbTe-Se:Na (band convergence), ${ }^{62} \mathrm{PbTe}-\mathrm{Mg}: \mathrm{Na}$ (band convergence), ${ }^{60}$ and PbTe:Tl (resonant level) $;^{64} \mathbf{d}$ graphic presentation of band alignment; e the ratio of carrier mobility to lattice thermal conductivity as a function of SrTe content in PbTe-Sr:Na; ${ }^{59}$ f temperature-dependent $Z T$ values of PbTe-Se:Na, PbTe-Mg:Na, PbTe:Tl, and PbTe$\mathrm{Sr}: \mathrm{Na}$

\section{OPTIMIZATIONS OF CARRIER DENSITY}

$\mathrm{PbTe}$ possesses the $\mathrm{NaCl}$-type crystal structure with space group of $F m-3 m$, which exhibits high symmetry. The unit cell is facecentered cube and all sites in the lattice have six coordinated atoms, as shown in Fig. 2a. The chemical bonds in PbTe are mixed ionic-covalent bonds, thus it is usually classified as polar semiconductor. The electronic band structure of PbTe in Fig. $2 \mathrm{~b}$ shows a typical semiconductor with a direct bandgap $\sim 0.3 \mathrm{eV} .{ }^{65}$ Distinct differences between valence band and conduction band can be easily observed, and the offset of light valence band $(L)$ and heavy valence band $(\Sigma)$ is $\sim 0.15-0.20 \mathrm{eV}^{69}$ while a larger band offset $\sim 0.45 \mathrm{eV}$ exists in conduction band. ${ }^{70}$

To obtain p-type PbTe, theoretically, alkali metal in group-IA ( $\mathrm{Li}$, $\mathrm{Na}, \mathrm{K}$ ) can work as an acceptor dopant in $\mathrm{Pb}$ site and produce hole carrier. Experimentally, most of the advanced p-type PbTe materials in Fig. $2 \mathrm{c}$ are doped with $\mathrm{Na}$ and $\mathrm{K}$, and the optimal hole carrier density is in the range of $3-40 \times 10^{19} \mathrm{~cm}^{-3}$. In n-type
PbTe, elements of I and $\mathrm{Br}$ (doping on Te site), $\mathrm{Sb}, \mathrm{Bi}, \mathrm{Al}, \mathrm{Ga}$, and In (doping on $\mathrm{Pb}$ sites) are usually chosen as donor dopants, and the electron carrier density in superior $\mathrm{n}$-type $\mathrm{PbTe}$ is in the range of $4-40 \times 10^{18} \mathrm{~cm}^{-3}$, shown in Fig. $2 \mathrm{~d}$. Notably, the optimal carrier density in p-type $\mathrm{PbTe}$ is an order of magnitude higher than that in n-type PbTe.

\section{MANIPULATIONS ON VALENCE BAND STRUCTURE IN P-TYPE PBTE}

In physics, quantum is the minimum unit to characterize physical properties that involve interaction. In thermoelectric materials, charge is a quantum unit of carriers that is used to estimate the electrical transport properties. Since the quantum charge transports in crystalline materials are closely relevant to electronic band structure, manipulation on electronic band structure is an effective strategy to optimize electrical transport properties. Manipulating approaches on electronic band structure include band 
convergence, DOS distortion and resonant state, band alignment between matrix and second phase, band flattening, and introducing impurity level, etc.

\section{Band convergence}

In p-type PbTe, the small offset between light valence band $(L)$ and heavy valence band $(\Sigma)$ facilitates charge redistribution from single band to multiple bands. Elements alloying could enclose the light and heavy valence bands, called band convergence. Converging valence bands could enhance effective mass through increasing valley degeneracy numbers. ${ }^{3,71}$ Recently, the approach of band convergence was revisited along with nanostructuring technology, which is one powerful synergistic strategy to enhance thermoelectric performance in p-type PbTe. ${ }^{62,72}$ Band convergence can achieve a high valley degeneracy number $\left(N_{\mathrm{v}}\right)$ to enlarge the effective mass $\left(m^{*}\right)$, defined as $m^{*}=N_{v}^{2 / 3} m_{\mathrm{b}}^{*}$, where $m_{\mathrm{b}}^{*}$ denotes the local band effective mass. Because the carrier mobility $\mu$ is proportional to $1 / m_{\mathrm{b}}^{*}$, the mobility is nominally unaffected by $N_{\mathrm{v}}$. Even though the carrier mobility may be deteriorated by the inter-valley scattering, the enhancement in effective mass overcomes the loss in carrier mobility, resulting in an enhanced net $Z T$.

It should be noted that the multiple electronic bands can be activated only when the band energy offsets are small enough and comparable to several $k_{\mathrm{B}} T$, where $k_{\mathrm{B}}$ is the Boltzmann constant. In p-type PbTe, the light $(L)$ and heavy $(\Sigma)$ bands in valence band are $\sim 0.15-0.20 \mathrm{eV}$, as shown in Fig. $3 \mathrm{a}$, and the small energy offset makes the multiple valence bands easily converge. Typically, several superior p-type PbTe-based systems are achieved through manipulating electronic band structures, and typical examples are $\mathrm{PbTe}-\mathrm{PbSe},{ }^{62} \mathrm{PbTe}^{-\mathrm{PbS}^{61,63}{ }^{6 b T e}-\mathrm{MgTe}^{60}}$

(a)

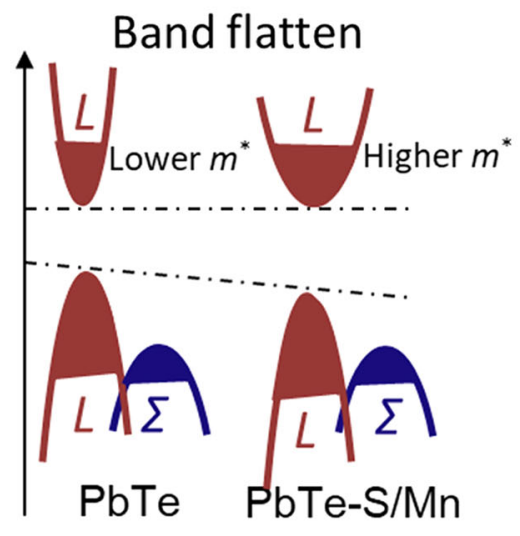

(c)

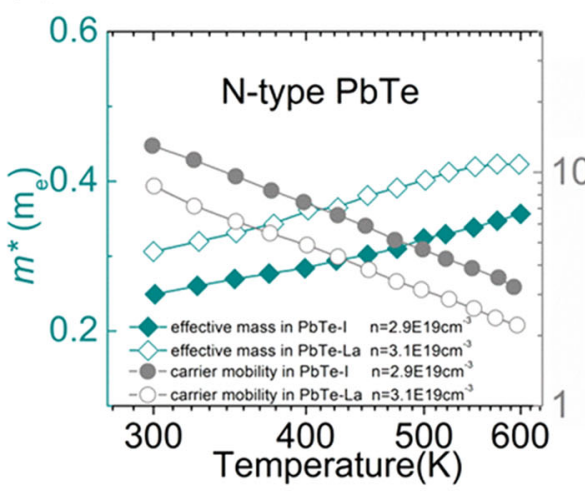

PbTe-SrTe 53,59 etc. In the valence bands of PbTe, valley degeneracy at $L$ point is 4 , while the valley degeneracy at $\Sigma$ point is $12 .^{62}$ Therefore, when two valence bands jointly carry carriers at a close energy range, the effective mass $m^{*}$ can get a dramatic enhancement, as shown in Fig. 3c. It is readily seen that the effective masses in PbTe-PbSe and PbTe-MgTe systems are larger than the Pisarenko line that is derived from the single parabolic band (SPB) model with an effective mass $m^{*}=0.3 m_{\mathrm{e}}$, evidencing a larger effective mass contributed by multiple valence bands.

\section{DOS distortion and resonant state}

Another alternative mechanism to increase effective mass and Seebeck coefficient is to produce distortion energy level into the conduction or valence bands. The effect of locally increased DOS to improve Seebeck coefficient can be explained by the Mott expression as follows: ${ }^{64}$

$$
\begin{aligned}
S & =\frac{\pi^{2} k_{B}^{2} T}{3 q}\left\{\frac{d[\ln (\sigma(E))]}{d E}\right\}_{E=E_{f}} \\
& =\frac{\pi^{2} k_{B}^{2} T}{3 q}\left\{\frac{1}{n} \frac{d n(E)}{d E}+\frac{1}{\mu} \frac{d \mu(E)}{d E}\right\}_{E=E_{f}}
\end{aligned}
$$

where $q$ is unit charge, $k_{\mathrm{B}}$ is Boltzmann constant, $\sigma(E)$ denotes energy-dependent electrical conductivity, $n$ denotes carrier density, $\mu$ is carrier mobility and $T$ is working temperature. From Eq. (1), we can understand that the enhancement in Seebeck coefficient induced by resonant level arises from two mechanisms: (1) an excess density of states $n(E)$ near Fermi level, and (2) increased energy-dependent $\mu(E)$, known as "resonant scattering". $^{73}$ The first mechanism is an intrinsic feature that is slightly temperature-dependent, and it will predominate with rising temperature. The second mechanism only works well at cryogenic

(b)

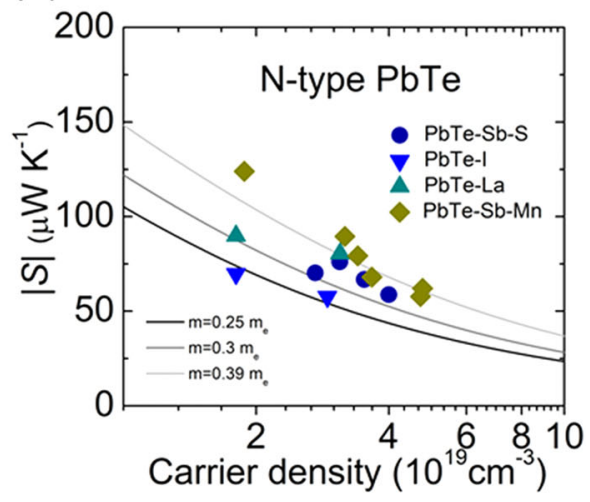

(d)

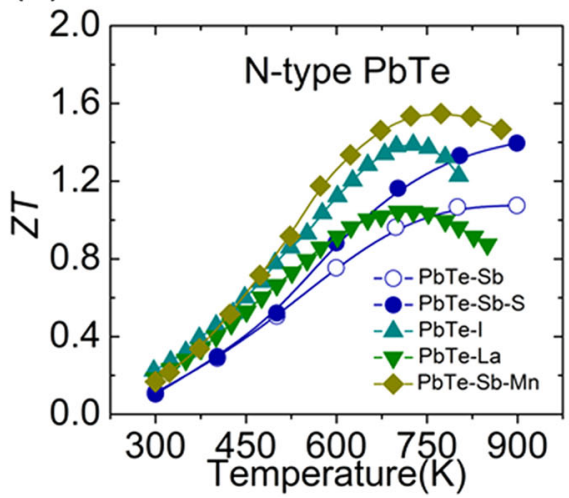

Fig. 4 a Schematic band flattening after $\mathrm{S}$ or Mn alloying in n-type PbTe; $\mathbf{b}$ Pisarenko line shows the different effective masses with doping and alloying; c comparisons of effective mass and carrier mobility for the I- and La-doped n-type PbTe; $\mathbf{d}$ temperature-dependent of $Z T$ values for I- and La-doped PbTe, n-type $\mathrm{PbTe}_{1-x} \mathrm{~S}_{x}$ and $\mathrm{Pb}_{1-x} \mathrm{Mn}_{x} \mathrm{Te}^{66,73,79}$ 
temperature when the phonon-electron scattering is weak. When phonon-electron scattering dominates in elevated temperature range, "resonant scattering" plays an ignorable role in enhancing Seebeck coefficient. Therefore, resonant state is very sensitive to temperature and it can only play a significant role in enhancing Seebeck coefficient near and below room temperature range. ${ }^{73}$ Fig. $3 \mathrm{~b}$ schematically presents the effects of resonant level on the electronic energy distribution in $\mathrm{PbTe}$ matrix, which could cause a sharp increase of DOS near Fermi level. This phenomenon has been observed in p-type Tl-doped PbTe system, ${ }^{51}$ and the $\mathrm{TI}$ resonant level in valence band of $\mathrm{PbTe}$ indeed contributes to a distinct increase of Seebeck coefficient shown in Fig. 3c. When coming to carrier mobility issue, it should be noted that DOS distortion will cause a larger carrier mobility deterioration than that caused by band convergence. The DOS distortion enhances the total effective mass through producing larger local band effective mass, however, the band convergence enhances the total effective mass through increasing valley degeneracy number.

Band alignment between PbTe matrix and nanostructured second phases

It is well-known that introducing defects in a host material not only intensifies phonon scattering, but also deteriorates carrier mobility. When nanostructured second phase exists in matrix, it is crucial to maintain high carrier mobility. One of the most effective approaches is to introduce the exotic phase with small band offset compared with matrix, namely band alignment. Actually, band alignment works very well in solar cell community to maintain carrier mobility, ${ }^{74,75}$ which was firstly well elucidated in PbTe system. ${ }^{65}$ Fig. $3 \mathrm{~d}$ schematically presents the function of band

(a)
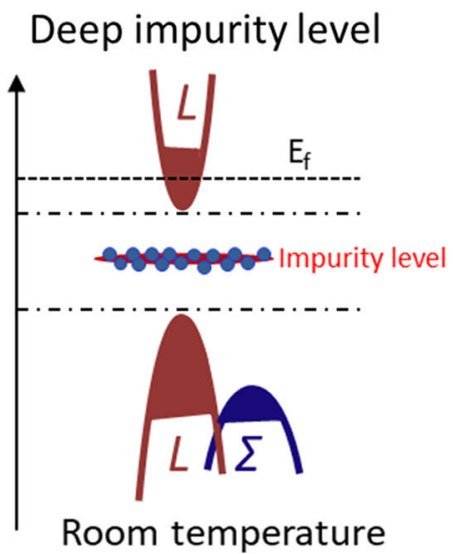

(c)

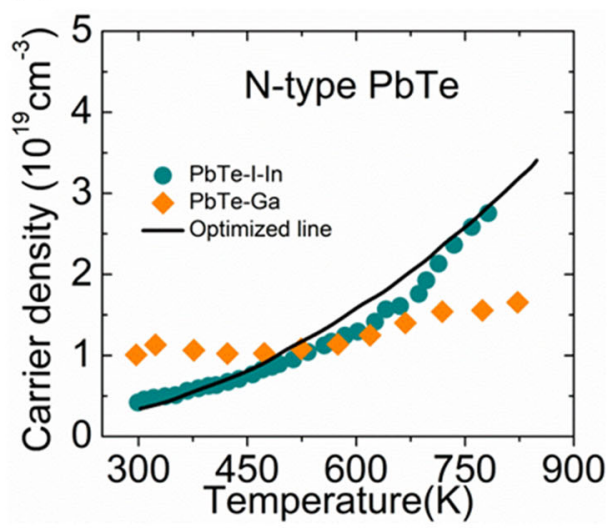

alignment in p-type PbTe-SrTe. ${ }^{65}$ Because the energy of valence band in $\mathrm{SrTe}$ is comparable to that of $\mathrm{p}$-type $\mathrm{PbTe}$, when second phase SrTe precipitates out in p-type PbTe matrix, the carrier mobility will be slightly deteriorated but phonon scattering is intensified by precipitates, which is considered as a typical example of well-known "phonon glass-electron crystal" proposed by Slack. ${ }^{76}$ To quantitatively evaluate the contribution of band alignment to thermoelectric performance, one can calculate the ratio of carrier mobility to lattice thermal conductivity $\left(\mu / \kappa_{\text {lat }}\right)$, as shown in Fig. 3e. The large increase in $\mu / \kappa_{\text {lat }}$ indicates that SrTe phases in p-type PbTe matrix scatter phonon stronger than carrier.

The final enhancements in $Z T$ values for p-type PbTe by synergistic approaches are well elucidated in Fig. $3 f$, which reveals that the methods of band convergence, resonant level and band alignment are pretty effective to enhance the electrical transport properties in p-type PbTe. More importantly, these approaches are also promising to be extensively applied in thermoelectric community as a general route.

\section{MANIPULATIONS ON CONDUCTION BAND STRUCTURE IN N- TYPE PBTE}

Flattening band structure

In n-type PbTe, conduction band flattening can distinctly enlarge the effective mass, thereby optimizing Seebeck coefficient. ${ }^{77}$ For an SPB, the local band effective mass $m_{b}^{*}$ near the band edge is sensitive to the band shape. From the energy dispersion

(b)

$$
\text { Deep impurity level }
$$

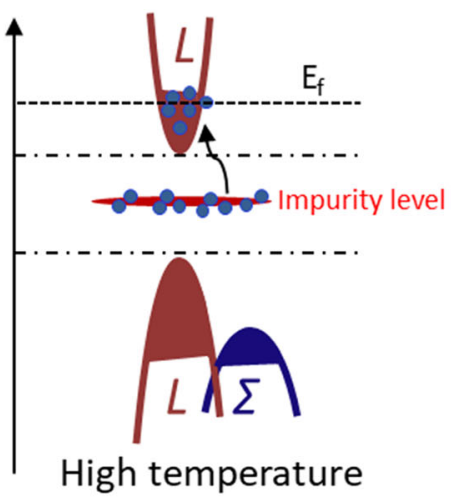

(d)

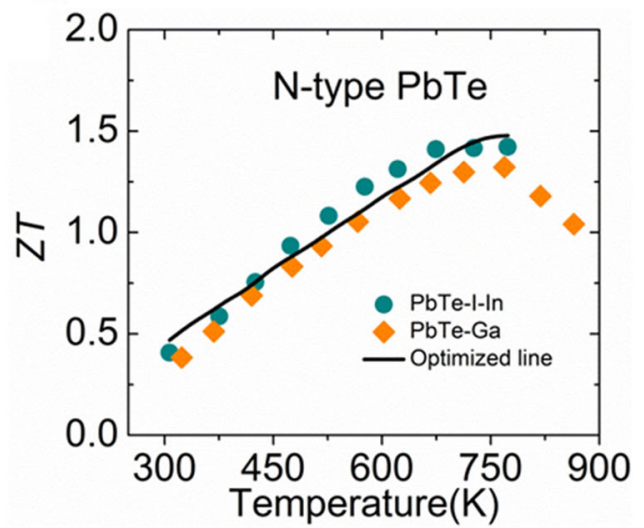

Fig. 5 Diagram schematically describes the deep impurity level in n-type PbTe: a deep impurity level localizes in bandgap and traps electrons at $300 \mathrm{~K}$; b deep impurity level releases electrons into conduction band at elevated temperature; c carrier density in both In- and Ga-doped $n-$ type $\mathrm{PbTe}$ increases with increasing temperature; d temperature-dependent $Z T$ values in In- and Ga-doped $\mathrm{n}$-type PbTe ${ }^{67,82}$ 
relationship, the $m_{\mathrm{b}}^{*}$ is defined by following equation:

$m_{\mathrm{b}}^{*}=\hbar^{2}\left(\frac{\partial^{2} E(k)}{\partial k^{2}}\right)^{-1}$

where $\hbar$ is reduced Planck constant, $E(\mathrm{k})$ and $\mathrm{k}$ denotes energy dispersion function and wave vector, respectively, in the reciprocal space. The Eq. (2) demonstrates flat band shape will lead to high effective mass, as shown in Fig. 4a. Experimentally, elements $S$ or $\mathrm{Mn}$ alloying in $\mathrm{n}$-type $\mathrm{PbTe}$ could produce an obvious band flattening and thereby an enhancement in carrier effective mass in Fig. 4b. Typically, the $Z T_{\max }$ values of n-type PbTe are largely boosted from $\sim 1.1$ to $\sim 1.4$ at $923 \mathrm{~K}$ and $\sim 1.6$ at $723 \mathrm{~K}$ through flattening conduction bands. ${ }^{66,77}$ In fact, as suggested by Kane band model, the energy dispersion is closely related to the bandgap $E_{\mathrm{g}}$, and broadening bandgap will flatten conduction band in PbTe following the relationship below: ${ }^{69,78}$

$\frac{\hbar^{2} k^{2}}{2 m_{b}^{*}}=E\left(1+\frac{E}{E_{g}}\right)$

It is noteworthy to point out that carrier mobility $\mu$ will also be inevitably deteriorated due to the relationship of $\mu \propto 1 / m_{b}{ }^{*}$ after conduction band flattening. Therefore, it should be careful to balance carrier effective mass and carrier mobility. Typically, combination of lower effective mass and higher carrier mobility in Fig. $4 \mathrm{C}$ leads to a high $Z T_{\max }$ value in I-doped PbTe $(\sim 1.4$ at $723 \mathrm{~K})$, which is $40 \%$ higher than that in La-doped PbTe $(\sim 1.0$ at $723 \mathrm{~K}){ }^{79}$ as shown in Fig. 4d.

\section{Deep impurity level}

Impurity levels caused by adding group-IIIA elements ( $\mathrm{Ga}$, In, TI) in $\mathrm{PbTe}$ are systematically investigated. ${ }^{80,81}$ At room temperature, Ga and In elements can from a deep impurity level in the forbidden bandgap. On the contrary, $\mathrm{Tl}$ impurity level will enter into the valence band to form a resonant level in p-type $\mathrm{PbTe}$, as we discussed above. ${ }^{64}$ Fig. 5a schematically describes the deep impurity level in n-type $\mathrm{PbTe}$ produced by introducing $\mathrm{Ga}$ and In. Interestingly, $\mathrm{Ga}$ and In $(+3)$ substitutions on $\mathrm{Pb}(+2)$ sites do not boost the carrier density of PbTe. At low temperature, the impurity levels produced by $\mathrm{Ga}$ and In could trap free electrons and work as a charge reservoir. ${ }^{67,82}$ With rising temperature, the trapped electrons will be released from the deep impurity levels into conduction band, finally increasing the carrier density as shown in Fig. 5b. In the n-type PbTe with In doping, the carrier density undergoes an order of magnitude enhancement from $\sim 5 \times 10^{18}$ $\mathrm{cm}^{-3}$ at $300 \mathrm{~K}$ to $\sim 2.8 \times 10^{19} \mathrm{~cm}^{-3}$ at $773 \mathrm{~K}$ in Fig. $5 \mathrm{c}$, exhibiting a kind of dynamic doping behavior. As for the PbTe with a single conduction band, this anomalous variation in carrier density is related to the changing charge states of $\mathrm{Ga}$ and $\mathrm{In}$. With increasing temperature, the mixed charge states $(+1$ and +3$)$ of $\mathrm{Ga}$ and In will change to fully +3 states. $^{80}$ Consequently, the dynamic doping extends $Z T_{\max }$ in a larger temperature range, resulting in a large average $Z T\left(Z T_{\text {ave }}\right) .^{67,82}$ The $Z T_{\text {ave }}$ over the entire working temperature range is important as it determines the thermoelectric conversion efficiency. ${ }^{22}$ Similarly, $\mathrm{Cr}^{83} \mathrm{Fe}^{84} \mathrm{Ti}^{85} \mathrm{Sc}^{86}$ etc. can introduce impurity states in $\mathrm{PbTe}$, however, these resonant impurity levels in conduction band are localized and contribute less to the carrier transports. ${ }^{85}$

\section{CHARGE TRANSPORTS IN PBTE-BASED SUPERLATTICE}

Theoretical prediction shows that low-dimensional materials such as superlattice thin films and nanowires can significantly enhance thermoelectric performance. ${ }^{7,9}$ Quantum confinement offers one special way to manipulate carrier transports because of the close relationship between electronic band structure and dimensionality. For the thermoelectric materials with quantum dots, the band offset and/or strain field between quantum dots and matrix (a)

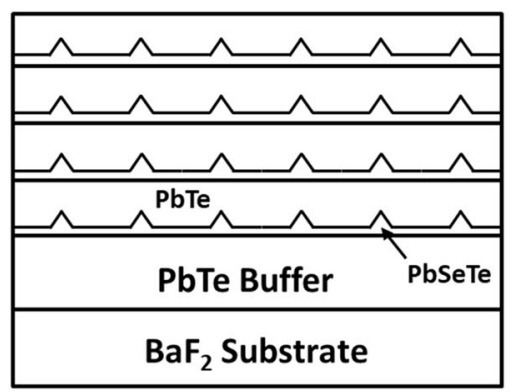

(c)

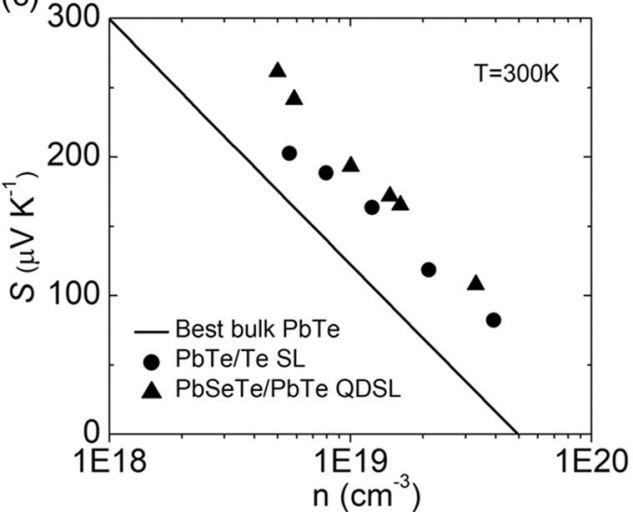

(b)

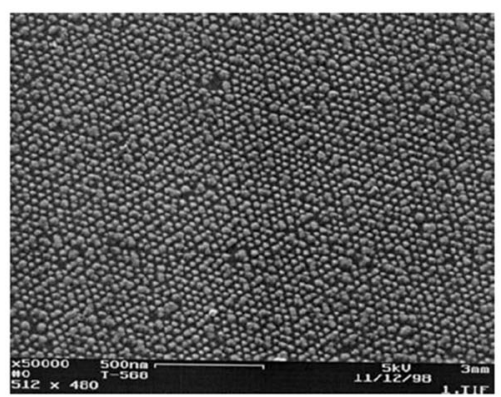

(d)

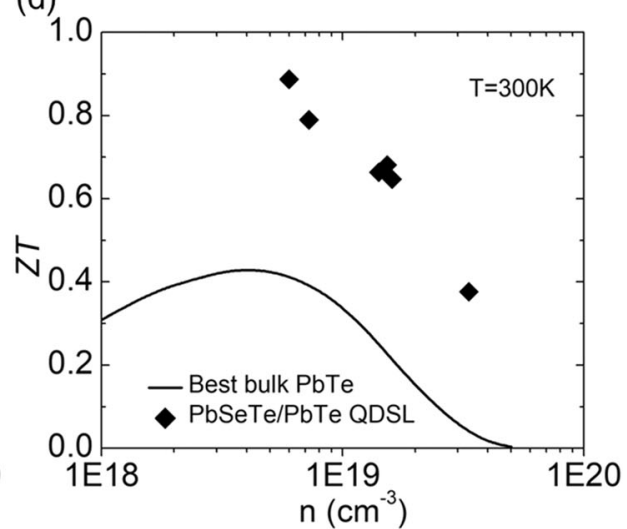

Fig. 6 a Schematic cross section of quantum-dot superlattice structure; $\mathbf{b}$ field-emission scanning electron microscope (FE-SEM) image of quantum-dot superlattice structure; ${ }^{87}$ c relationship between Seebeck coefficient and carrier density for bulk PbTe and the new PbTe-based quantum-dot superlattice structures; d comparison of $Z T$ as a function of carrier density between bulk $\mathrm{PbTe}$ and $\mathrm{PbSeTe} / \mathrm{PbTe}$ quantum-dot superlattice structures (data taken from ref. ${ }^{87}$ ) (b adapted from ref. ${ }^{87}$ with permission, copyright Springer Nature 2000) 

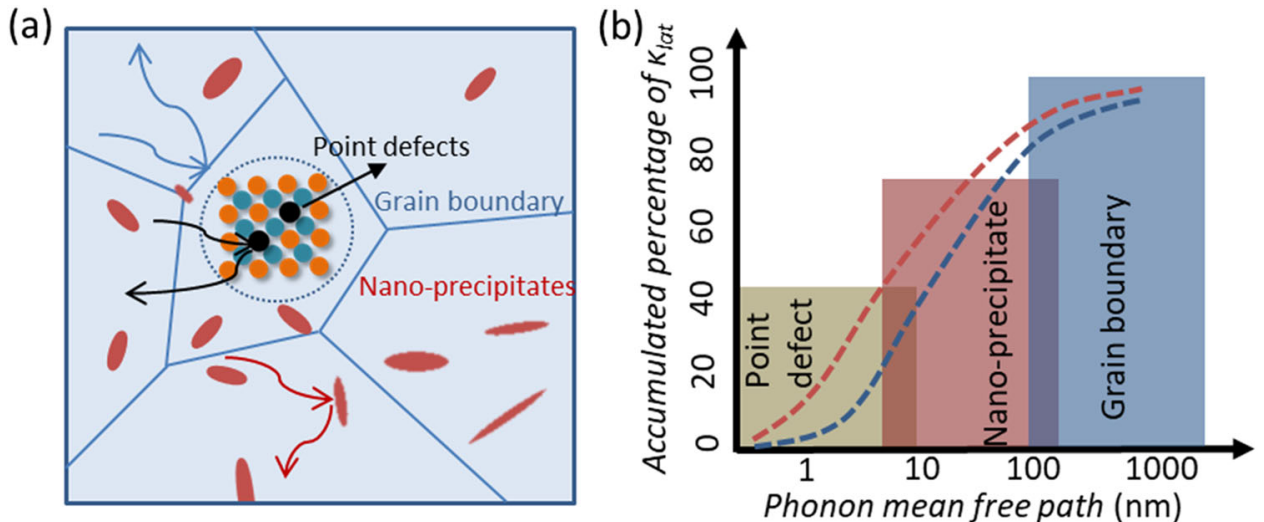

Fig. 7 a Diagram schematically describes all-scale hierarchical architecture to scatter phonon; $\mathbf{b}$ accumulated contributions to reduce lattice thermal conductivity with respect of phonon mean free path in PbTe
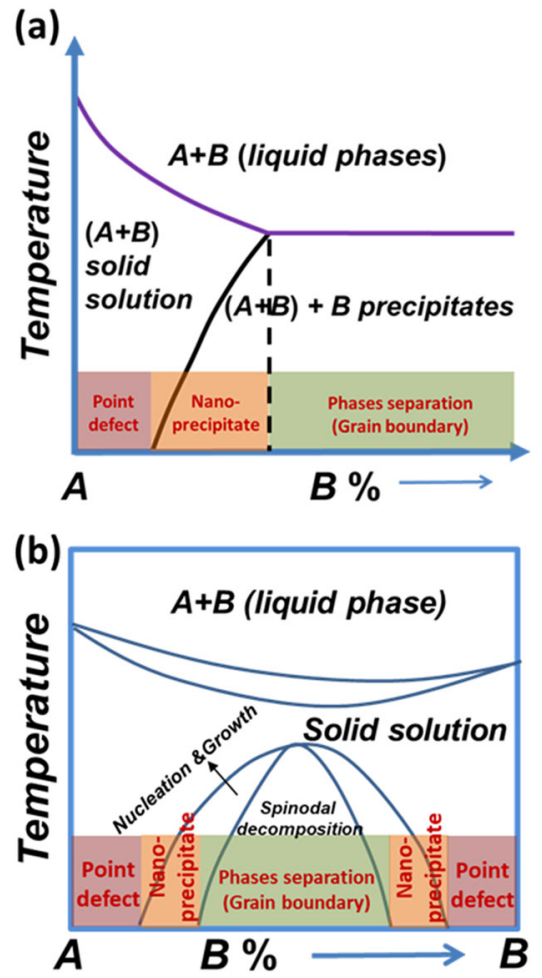

Fig. 8 Phase diagram to guide all-scale hierarchical architecture: a eutectic phase diagram; b spinodal decomposition diagram. The targeted microstructures (right inserts) can be obtained if the synthesis process in given composition can be well controlled

could act as a confining potential. Therefore, the electronic density of states $(d N / d E)$ has the shape of a Dirac delta function ${ }^{87}$

$d N / d E \propto \sum_{\varepsilon_{i}} \delta\left(E-\varepsilon_{i}\right)$

where $\varepsilon_{\mathrm{i}}$ is discrete energy levels and $\delta$ is the Dirac function. Figure $6 a$ schematically shows the $\mathrm{PbSeTe} / \mathrm{PbTe}$ superlattice structures prepared by molecular beam epitaxy (MBE). The field emission scanning electron microscope (FE-SEM) in Fig. $6 \mathrm{~b}$ shows individual quantum dots in white massively distribute in planar arrays. Compared with the state-of-the-art PbTe bulks, the artificial superlattice structures could produce much larger Seebeck coefficient in Fig. 6c. The increased Seebeck coefficient arises from larger effective mass caused by the band structure modification due to quantum confinement. Figure $6 \mathrm{~d}$ elucidates that the $Z T_{\max }$ value can be enhanced through designing PbSeTe/ $\mathrm{PbTe}$ superlattice, indicating that reducing dimensionality of a given material is a potential possibility to enhance thermoelectric performance.

\section{MANIPULATIONS ON PHONON TO REDUCE LATTICE THERMAL CONDUCTIVITY}

Apart from electrical transport properties, low thermal conductivity is required to preserve large temperature gradient, which is a vital factor to realize high thermoelectric conversion efficiency. Heat transport is regarded as elementary vibrational motion, and the atom oscillation can be quantitatively evaluated using a quantum description "phonon". Phonon propagation and dispersion in crystalline materials are closely related to the mean free path (MFP), which ranges from atomic-scale, nano-scale to mesoscale. Since the MFP of PbTe is predominated in nano-scale, therefore, nanostructuring is widely applied to reduce lattice thermal conductivity in PbTe. ${ }^{16,59}$ When the defect sizes are comparable to the MFP range, the phonon scattering can be dramatically intensified and therefore lead to a very low lattice thermal conductivity. ${ }^{54,55}$ Based on this idea, artificially designing defects with all-scale hierarchical architectures are put forward to considerably reduce lattice thermal conductivity.

Figure $7 a$ is a schematic description of all-scale hierarchical structures including atomic-scale point defects, nanoscale precipitates and mesoscale grain boundaries. The contributions of reduced lattice thermal conductivity from these all-scale defects can be quantitatively estimated by: ${ }^{88,89}$

$\kappa_{l}=\frac{k_{\mathrm{B}}}{2 \pi^{2} \nu}\left(\frac{k_{\mathrm{B}} T}{\hbar}\right)^{3} \int_{0}^{\theta_{\mathrm{D}} / T} \tau_{\mathrm{c}}(x) \frac{x^{4} e^{x}}{\left(e^{x}-1\right)^{2}} \mathrm{~d} x$

where $v$ is average phonon-group velocity, $T$ is working temperature, $\theta_{\mathrm{D}}$ is Debye temperature, $x=h \omega / \kappa_{\mathrm{B}} T$, and $\tau$ is relaxation time. Here, the relaxation time is treated as an integration of various process including Umklapp process $\left(\tau_{\mathrm{U}}\right)$, normal process $\left(\tau_{\mathrm{N}}\right)$, grain boundary process $\left(\tau_{\mathrm{G}}\right)$, nano-precipitate process $\left(\tau_{\mathrm{NP}}\right)$ and point defect process $\left(\tau_{\mathrm{PD}}\right): 89,90$

$\frac{1}{\tau_{\mathrm{C}}}=\frac{1}{\tau_{\mathrm{U}}}+\frac{1}{\tau_{\mathrm{N}}}+\frac{1}{\tau_{\mathrm{G}}}+\frac{1}{\tau_{\mathrm{NP}}}+\frac{1}{\tau_{\mathrm{PD}}}$

When all above mechanisms contribute to the scattering of phonon propagation, the lattice thermal conductivity is accumulatively suppressed as shown in Fig. 7b. The lattice thermal conductivity can be described as $\kappa_{l}=1 / 3 C \mathrm{Cl}$, where $C$ denotes heat capacity per unit volume, $v$ is average speed of lattice vibration, $/$ is mean free path. When / reaches interatomic distance, the lattice thermal conductivity can approach the theoretical 


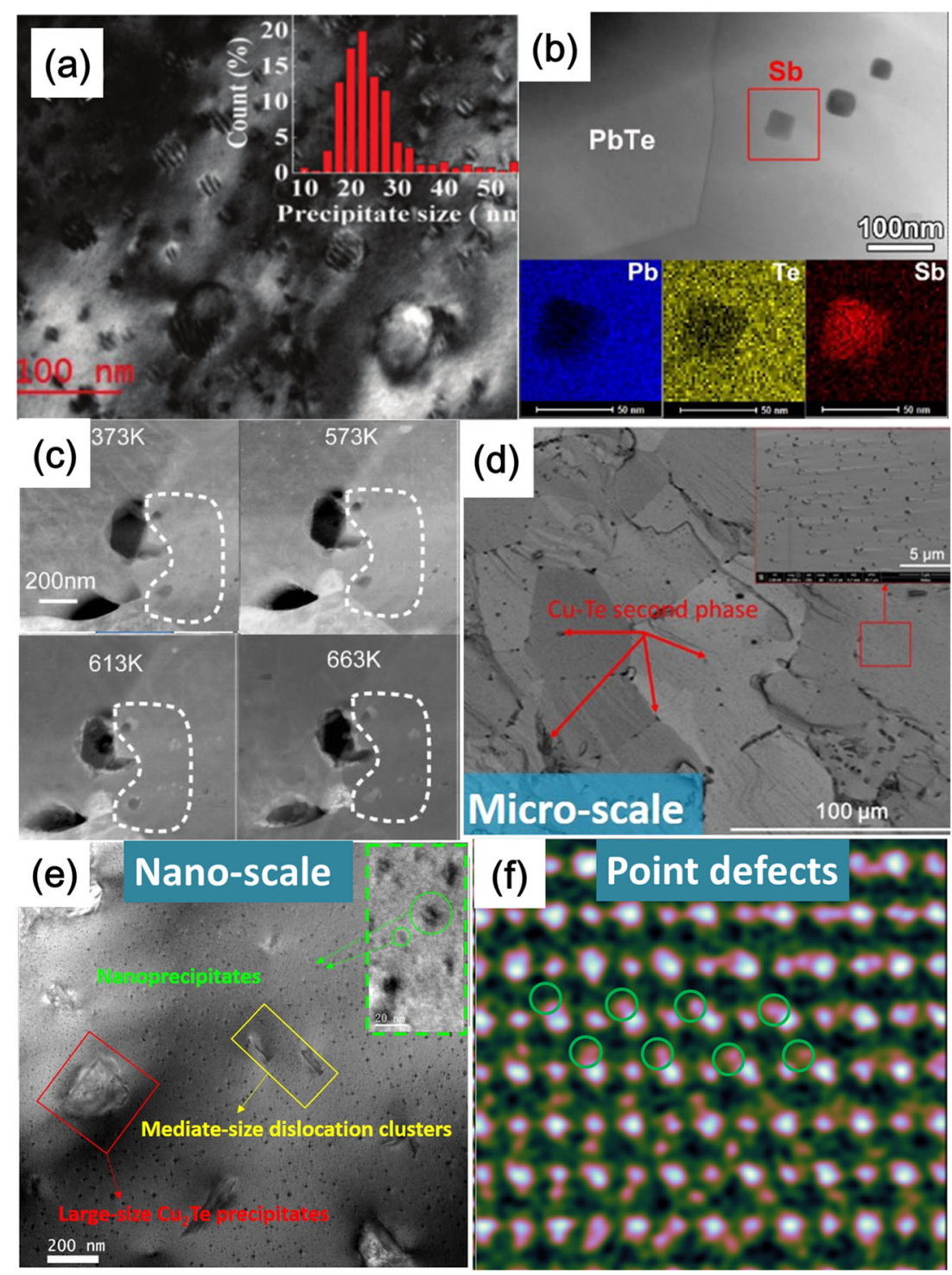

Fig. 9 a TEM image of nano-precipitates with size distribution histogram as the inset in PbTe-4\% $/ n S b ;{ }^{54} \mathbf{b}$ the HADDF image of Sb precipitates in the $\mathrm{PbTe}_{0.998} \mathrm{l}_{0.002}-3 \% \mathrm{Sb}$, the insets are the EDS elemental maps of one selected precipitates; $\mathbf{c}$ the in suit HADDF images of Sb precipitates in the $\mathrm{PbTe}_{0.998} \mathrm{l}_{0.002}-3 \% \mathrm{Sb}$ with temperature rising from $373 \mathrm{~K}$ to $663 \mathrm{~K}_{i}^{.55}$ microstructure observation in n-type $\mathrm{PbTe}$-Cu $\mathrm{Te}_{2} \mathrm{Tample:} \mathbf{d}$ grain boundary in mesoscale; e precipitates in nanoscale; $\mathbf{f}$ point defects of Cu interstitial. ${ }^{98}$ (a adapted from ref. ${ }^{54}$ with permission, copyright John Wiley and Sons 2017) (b and c adapted from ref. ${ }^{55}$ with permission, copyright Royal Society of Chemistry 2017) (d, e, and f adapted from ref. ${ }^{98}$ with permission, copyright American Chemical Society 2017)

minimum $\kappa_{\min }$. It should be pointed out that the MFP range is related to the lattice-vibration frequency, which is temperaturedependent, implying that more scattering mechanisms may be involved in a given temperature.

Usually, nano-precipitates experience continuous steps of nucleation and growth, thus nano-precipitates range from several nanometers to hundreds of nanometers. Both eutectic phase diagram and spinodal decomposition phase diagram in Fig. 8 show two representative ways to obtain nanostructures $B$ in $A$ matrix. In Fig. 8a, the $A$ and $B$ will be homogeneously melting at high temperature, when $B$ exceeds its solubility limit in $A$, it will precipitate out as nanostructures, which are well elucidated in PbTe-MTe ( $\mathrm{M}=\mathrm{Mg}, \mathrm{Ba}, \mathrm{Sr}, \mathrm{Ca}$ and $\mathrm{Cd}$ ) systems..$^{59,60,91-93}$ Fig. 8b exhibits a miscibility gap where thermodynamic phase separation will occur through metastable nucleation and growth, which is called spinodal decomposition process. Spinodal decomposition can also produce different nano-precipitates, which is similar to that of eutectic process, as shown in the right column of Fig. 8.
Regardless eutectic phase diagram and spinodal decomposition, when $A$ and $B$ possess similar crystal structure and comparable lattice parameter, a coherent or semi-coherent interface between matrix and nano-precipitates will be formed, which will reduce the carrier scattering and facilitate carrier transport between phase interfaces. $^{94}$ The nano-precipitates with coherent or semicoherent interfaces have been widely observed in PbTe-PbQ (Q $=\mathrm{Se}, \mathrm{S}$ ) solid solutions. ${ }^{61,63,95,96}$ Interestingly, the shapes and sizes of nano-precipitates in a given thermoelectric system are also sensitive to the processing parameters during synthesizing, such as cooling rate, annealing temperature and annealing time, etc. $^{53,97}$

Internal structure designs in PbTe-based thermoelectric materials Most of superior PbTe-based thermoelectric materials are obtained by reducing lattice thermal conductivity through scattering phonons via designing special microstructures. To match the high-performance p-type $\mathrm{PbTe}$, the approach of 
(a)

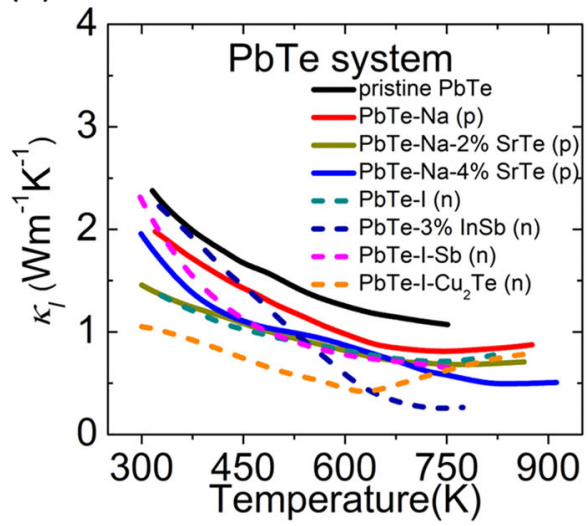

(b)

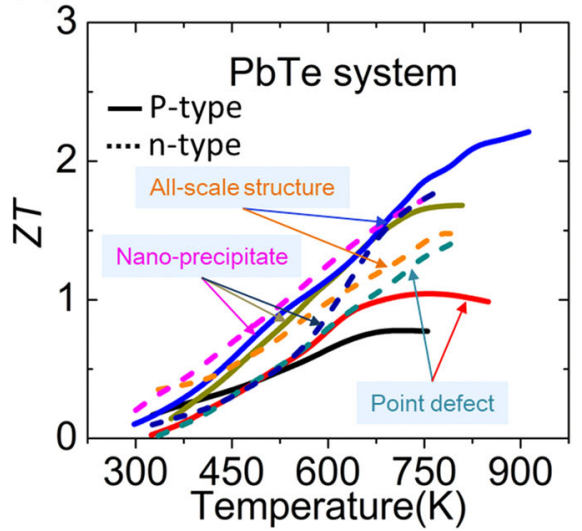

Fig. 10 a Temperature dependence of lattice thermal conductivity; b temperature dependence of $Z T$ values in PbTe with the all-scale hierarchical architecture

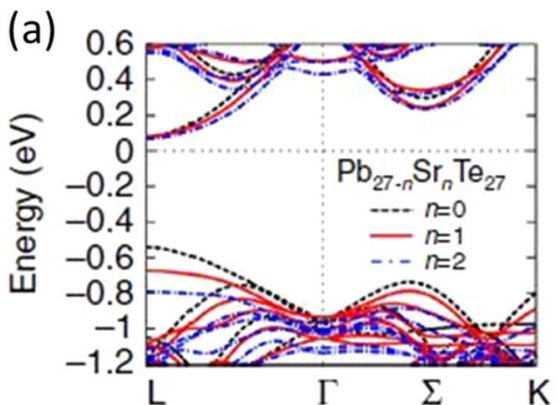

(c)

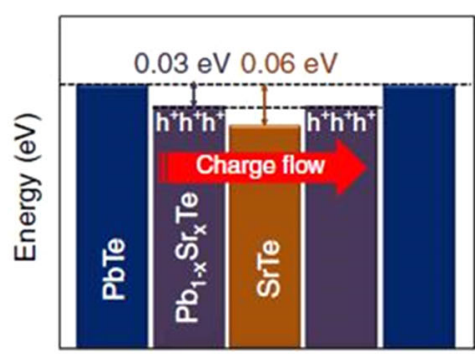

Valence band maximum (VBM)

(e)

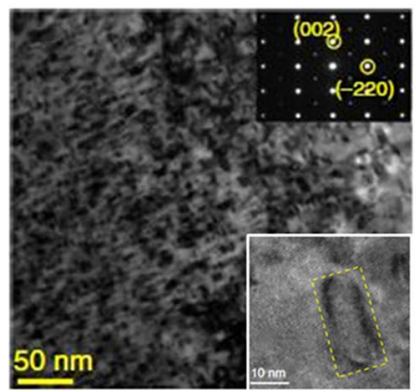

(b)

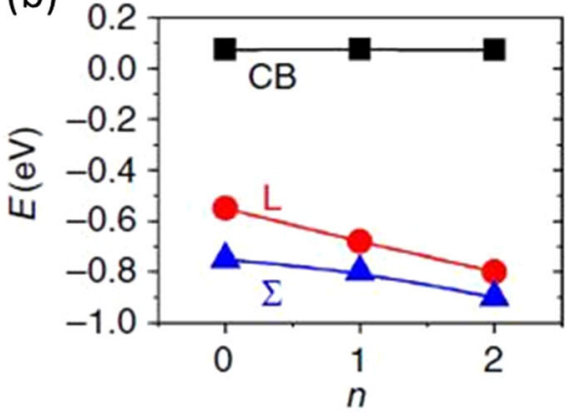

(d)

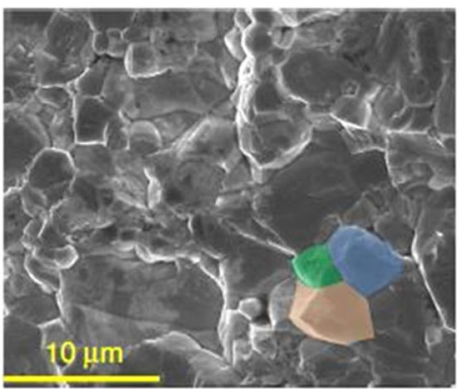

(f)

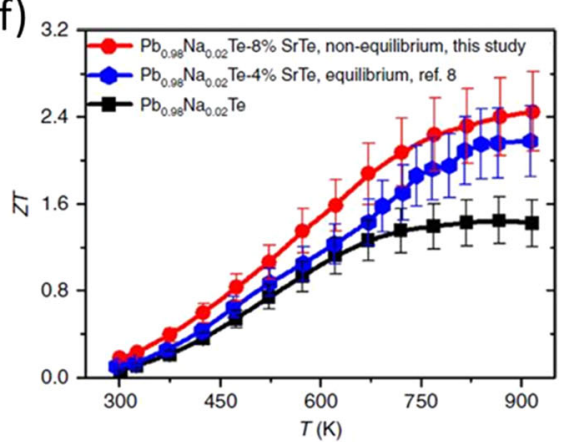

Fig. 11 Synergistic strategy to obtain high thermoelectric performance in PbTe-SrTe: a the band structure changing with varying SrTe fraction in $\mathrm{PbTe}$; $\mathbf{b}$ the relative band positions of valence band at $L, \Sigma$ point, and the conduction band; $\mathbf{c}$ band alignment between SrTe second phase and $\mathrm{PbTe}$ matrix to facilitate carrier transport; $\mathbf{d}$ SEM image showing micro-size grains; e low magnification TEM image showing ubiquitous nano-precipitates in PbTe matrix; $\mathbf{f}$ the $Z T$ values as a function of temperature in PbTe-SrTe. ${ }^{53}\left(\mathbf{a}, \mathbf{b}, \mathbf{c}, \mathbf{d}, \mathbf{e}\right.$, and $\mathbf{f}$ adapted from ref. ${ }^{53}$ with permission, copyright Springer Nature 2016) 
designing microstructures in n-type PbTe is highly sought since conduction bands are less complex than its valence bands. Recently, a record high $Z T_{\max } \sim 1.8$ at $773 \mathrm{~K}$ was obtained in n-type $\mathrm{PbTe}-\mathrm{InSb}$ with special nanostructures, ${ }^{54}$ as shown in Fig. 9a. Both electrical and thermal transport properties are improved in nanostructured n-type PbTe. Seebeck coefficients are enhanced due to carriers filtering by the energy barrier caused by nanostructures, which also contribute to extremely low lattice thermal conductivity due to strong phonon scattering. ${ }^{54} \mathrm{Sb}$ doping in n-type PbTe (Fig. 9b) also exhibits very interesting behaviors. Sb-contained endotaxial nano-precipitates can be clearly observed at room temperature, but $\mathrm{Sb}$ will dissolve into $\mathrm{PbTe}$ matrix with rising temperature as shown in Fig. $9 \mathrm{c}^{55}$ The solubility limit of $\mathrm{Sb}$ in $\mathrm{PbTe}$ is temperature-dependent, which could produce the dynamic doping and phonon scattering, thus both electrical and thermal transport properties are improved. ${ }^{55}$ The idea of all-scale hierarchical architectures is confirmed as an effective approach to enhance thermoelectric performance in ptype $\mathrm{PbTe}-\mathrm{SrTe}$ through reducing lattice thermal conductivity. ${ }^{59}$ The hierarchical architectures could also be realized in n-type $\mathrm{PbTe}$ just through introducing $\mathrm{Cu}^{98}$ as shown in Fig. $9 d-f$. The lattice thermal conductivity could be reduced as low as $\sim 0.38 \mathrm{Wm}^{-1} \mathrm{~K}^{-1}$ in PbTe-5.5\% $\mathrm{Cu}_{2} \mathrm{Te}$. Interestingly, as shown in Fig. $9 f$, massive $\mathrm{Cu}$ interstitials would induce local lattice distortion. Moreover, apart from the hierarchical architecturing, these $\mathrm{Cu}$ interstitials are inclined to vibrate in a large displacement, which could further suppress the lattice thermal conductivity.

Figure 10 shows the temperature-dependent lattice thermal conductivity and $Z T$ values in several typical PbTe systems. From this comparison, it is obviously seen that the sample with all-scale defects possesses the lowest lattice thermal conductivity, which is comparable to the theoretical minimum value $\left(\kappa_{\min } \sim\right.$ $0.36 \mathrm{Wm}^{-1} \mathrm{~K}^{-1}$ ) in Fig. 10a. The $Z T_{\max }$ value achieves $\sim 2.2$ at $915 \mathrm{~K}$ in p-type PbTe-Na-4\%SrTe and $\sim 1.8$ at $773 \mathrm{~K}$ in n-type $\mathrm{PbTe}_{0.998} \mathrm{l}_{0.002}-3 \% \mathrm{Sb}$, as shown in Fig. 10b. ${ }^{55,59}$

\section{PARADIGMS OF SYNERGIC STRATERGIES TO HIGH THERMOELECTRIC PERFORMANCE}

Here we introduce an interesting high performance p-type PbTeSrTe, in which the charge and phonon transports have been well manipulated using synergic strategies. ${ }^{53}$ The DFT calculation in Fig. 11a reveals that SrTe can effectively enlarge the bandgap and concurrently converge two valence bands. As shown in Fig. 11b, the light conduction band keeps at a fixed position, while the offset between two valence bands is reduced with increasing SrTe fraction. The valence band convergence contributes to a large Seebeck coefficient, and the enlarged bandgap can suppress the bipolar diffusion. In addition, carrier mobility is slightly deteriorated by SrTe precipitates due to valence band alignment between SrTe and PbTe, as shown in Fig. 11c. Furthermore, a special non-equilibrium synthesis process is used in PbTe-SrTe with ice water quenching, which produces small grains with size ranging from 1 to $3 \mu \mathrm{m}$ in Fig. $11 \mathrm{~d}$. Meanwhile, nano-precipitates are ubiquitously observed in Fig. 11e. Through using synergistically optimizing charge and phonon transports, a record high $Z T_{\text {max }}$ value $\sim 2.5$ can be realized in PbTe-8\%SrTe, as shown in Fig. $11 \mathrm{f}$. The synergistic strategy is also successfully used in PbTe$\mathrm{MgTe}^{60}{ }^{6 \mathrm{PbS}-\mathrm{CdS},}{ }^{18} \mathrm{SnTe}^{-C d T e},{ }^{99}$ (Ti, Zr)NiSn, ${ }^{100}$ etc. These cases indicate that synergistically manipulating charge and phonon transports is promising to be extended to other thermoelectric materials.

\section{SUMMARY AND OUTLOOK}

In the past decades, the emergence of new strategies enriched the story of $\mathrm{PbTe}$-based thermoelectric materials. A high performance can be realized in $\mathrm{p}$-type $\mathrm{PbTe}$-SrTe system through synergistically manipulating charge and phonon transports. One can see that most reports focused on p-type PbTe, but few reports on n-type $\mathrm{PbTe}$, which is urgent to be developed to match its high performance $\mathrm{p}$-type counterpart. The high performance $\mathrm{n}$-type $\mathrm{PbTe}$ is expected to be realized through using following approaches: obtaining higher carrier mobility through reducing intrinsic vacancies; scattering all-length phonons through designing all-scale hierarchical architectures; facilitating charge transports through aligning conduction bands; suppressing the bipolar diffusion through enlarging bandgap; enhancing effective mass through tailoring conduction band structures.

\section{ACKNOWLEDGEMENTS}

This work was supported by National Key Research and Development Program of China under Grant No. 2018YFB0703600, National Natural Science Foundation of China under Grant Nos. (51772012, 51571007 and 51632005), the Beijing Municipal Science \& Technology Commission under Grant No. Z171100002017002, 111 Project (B17002) and the Academic Excellence Foundation of BUAA for PhD Students. The authors are grateful to Professors M.G. Kanatzidis, C. Wolverton, D.P. Vinayak, C. Uher, J.-F. Li, J.Q. He, L. Huang, G.J. Tan, S.Q. Hao, H.J. Wu, S.J. Pennycook, Y.S. Zhang, S.H. Lo, $\mathrm{H}$. Sun, and $\mathrm{H}$. Chi for their plentiful discussions and fruitful collaborations.

\section{AUTHOR CONTRIBUTIONS}

Y.X. and L.-D.Z. drafted this perspective. L.-D.Z. offered important idea of the figures. Y.X. created the central idea of this review, constructed this framework. Both authors contributed to the writing of the manuscript.

\section{ADDITIONAL INFORMATION}

Competing interests: The authors declare no competing interests.

Publisher's note: Springer Nature remains neutral with regard to jurisdictional claims in published maps and institutional affiliations.

\section{REFERENCES}

1. DiSalvo, F. J. Thermoelectric cooling and power generation. Science $\mathbf{2 8 5}$, 703-706 (1999).

2. Tan, G., Zhao, L.-D. \& Kanatzidis, M. G. Rationally designing high-performance bulk thermoelectric materials. Chem. Rev. 116, 12123-12149 (2016).

3. Ravich, Y. I. et al. Semiconducting Lead Chalcogenides. (Plenum Press, 1970).

4. Vedernikov, M. V. \& lordanishvili, E. K. in Seventeenth International Conference on Thermoelectrics 37-42 (IEEE, 1998).

5. Kanatzidis, M. G. Nanostructured thermoelectrics: the new paradigm? Chem. Mater. 22, 648-659 (2010).

6. Heremans, J. P., Dresselhaus, M. S., Bell, L. E. \& Morelli, D. T. When thermoelectrics reached the nanoscale. Nat. Nanotechnol. 8, 471-473 (2013).

7. Hicks, L. \& Dresselhaus, M. S. Effect of quantum-well structures on the thermoelectric figure of merit. Phys. Rev. B 47, 12727 (1993)

8. Dresselhaus, M. S. et al. New directions for low-dimensional thermoelectric materials. Adv. Mater. 19, 1043-1053 (2007).

9. Hicks, L. D. \& Dresselhaus, M. S. Thermoelectric figure of merit of a onedimensional conductor. Phys. Rev. B 47, 16631-16634 (1993).

10. Zhang, X. \& Pei, Y. Manipulation of charge transport in thermoelectrics. npj Quant. Mater. 2, 68 (2017).

11. Mao, J., Liu, Z. \& Ren, Z. Size effect in thermoelectric materials. npj Quant. Mater. 1, 16028 (2016).

12. Kim, S. I. et al. Dense dislocation arrays embedded in grain boundaries for highperformance bulk thermoelectrics. Science 348, 109-114 (2015).

13. Poudel, B. et al. High-thermoelectric performance of nanostructured bismuth antimony telluride bulk alloys. Science 320, 634-638 (2008).

14. $\mathrm{Hu}, \mathrm{L}$. et al. Tuning multiscale microstructures to enhance thermoelectric performance of $\mathrm{n}$-type bismuth-telluride-based solid solutions. Adv. Energy Mater. 5, 1500411 (2015).

15. Zhao, $\mathrm{H}$. et al. High thermoelectric performance of MgAgSb-based materials. Nano Energy 7, 97-103 (2014).

16. Hsu, K. F. et al. Cubic $\mathrm{AgPb}_{\mathrm{m}} \mathrm{SbTe}_{2+\mathrm{m}}$ : bulk thermoelectric materials with high figure of merit. Science 303, 818-821 (2004).

17. LaLonde, A. D., Pei, Y., Wang, H. \& Jeffrey Snyder, G. Lead telluride alloy thermoelectrics. Mater. Today 14, 526-532 (2011). 
18. Zhao, L.-D. et al. Raising the thermoelectric performance of $\mathrm{p}$-type $\mathrm{PbS}$ with endotaxial nanostructuring and valence-band offset engineering using $\mathrm{CdS}$ and ZnS. J. Am. Chem. Soc. 134, 16327-16336 (2012).

19. Zhao, L.-D. et al. Thermoelectrics with earth abundant elements: high performance p-type PbS nanostructured with SrS and CaS. J. Am. Chem. Soc. 134, 7902-7912 (2012).

20. He, J. et al. Strong phonon scattering by layer structured PbSnS2 in PbTe based thermoelectric materials. Adv. Mater. 24, 4440-4444 (2012).

21. Zhao, L.-D. et al. Ultralow thermal conductivity and high thermoelectric figure of merit in SnSe crystals. Nature 508, 373-377 (2014).

22. Zhao, L.-D. et al. Ultrahigh power factor and thermoelectric performance in hole-doped single-crystal SnSe. Science 351, 141-144 (2016).

23. $\mathrm{Wu}, \mathrm{H}$. et al. Sodium-doped tin sulfide single crystal: a nontoxic earth-abundant material with high thermoelectric performance. Adv. Energy Mater. 8, 1800087 (2018).

24. Chang, $C$. et al. 3D charge and $2 D$ phonon transports leading to high out-ofplane ZT in n-type SnSe crystals. Science 360, 778-783 (2018),

25. Chen, Z.-G., Shi, X., Zhao, L.-D. \& Zou, J. High-performance SnSe thermoelectric materials: Progress and future challenge. Prog. Mater. Sci. 97, 283-346 (2018).

26. Zhou, Y. \& Zhao, L.-D. Promising thermoelectric bulk materials with 2D structures. Adv. Mater. 29, 1702676 (2017).

27. Zhao, L.-D., Chang, C., Tan, G. \& Kanatzidis, M. G. SnSe: a remarkable new thermoelectric material. Energy Environ. Sci. 9, 3044-3060 (2016).

28. Zhao, L.-D. et al. Enhanced thermoelectric properties in the counter-poped SnTe system with strained endotaxial SrTe. J. Am. Chem. Soc. 138, 2366-2373 (2016).

29. Zhang, Q. et al. High thermoelectric performance by resonant dopant indium in nanostructured SnTe. Proc. Natl Acad. Sci. USA 110, 13261-13266 (2013).

30. Wu, D. et al. Origin of the high performance in GeTe-based thermoelectric materials upon $\mathrm{Bi}_{2} \mathrm{Te}_{3}$ doping. J. Am. Chem. Soc. 136, 11412-11419 (2014).

31. Liu, H. L. et al. Copper ion liquid-like thermoelectrics. Nat. Mater. 11, 422-425 (2012).

32. He, Y. et al. Ultrahigh thermoelectric performance in Mosaic crystals. Adv. Mater 27, 3639-3644 (2015).

33. Liu, W. et al. Convergence of conduction bands as a means of enhancing thermoelectric performance of n-type $\mathrm{Mg}_{2} \mathrm{Si}_{1-x} \mathrm{Sn}_{\mathrm{x}}$ solid solutions. Phys. Rev. Lett. 108, 166601 (2012).

34. Liu, W. et al. n-type thermoelectric material $\mathrm{Mg}_{2} \mathrm{Sn}_{0.75} \mathrm{Ge}_{0.25}$ for high power generation. Proc. Natl Acad. Sci. USA 112, 3269-3274 (2015).

35. Zhao, L.-D. et al. BiCuSeO oxyselenides: new promising thermoelectric materials. Energy Environ. Sci. 7, 2900-2924 (2014).

36. Zhang, X., Chang, C., Zhou, Y. \& Zhao, L.-D. BiCuSeO thermoelectrics: an update on recent progress and perspective. Materials 10, 198 (2017).

37. Snyder, G. J., Christensen, M., Nishibori, E., Caillat, T. \& Iversen, B. B. Disordered zinc in $\mathrm{Zn}_{4} \mathrm{Sb}_{3}$ with phonon-glass and electron-crystal thermoelectric properties. Nat. Mater. 3, 458-463 (2004).

38. Rhyee, J.-S. et al. Peierls distortion as a route to high thermoelectric performance in $\ln _{4} \mathrm{Se}_{3-\delta}$ crystals. Nature 459, 965-968 (2009).

39. Toberer, E. S., Christensen, M., Iversen, B. B. \& Snyder, G. J. High temperature thermoelectric efficiency in $\mathrm{Ba}_{8} \mathrm{Ga}_{16} \mathrm{Ge}_{30}$. Phys. Rev. B 77, 075203 (2008).

40. Shi, X. et al. Multiple-filled skutterudites: high thermoelectric figure of merit through separately optimizing electrical and thermal transports. J. Am. Chem. Soc. 133, 7837-7846 (2011).

41. Meng, X. et al. Grain boundary engineering for achieving high thermoelectric performance in n-type skutterudites. Adv. Energy Mater. 7, 1602582 (2017).

42. Liu, W. S., Zhang, B. P., Li, J. F. \& Zhao, L.-D. Effects of Sb compensation on microstructure, thermoelectric properties and point defect of $\mathrm{CoSb} 3$ compound. J. Phys. D: Appl. Phys. 40, 6784-6790 (2007).

43. Lu, X. et al. High performance thermoelectricity in earth-abundant compounds based on natural mineral tetrahedrites. Adv. Energy Mater. 3, 342-348 (2013)

44. $\mathrm{Yu}, \mathrm{B}$. et al. Enhancement of thermoelectric properties by modulation-doping in silicon germanium alloy nanocomposites. Nano Lett. 12, 2077-2082 (2012).

45. Cheikh, D. et al. Praseodymium telluride: a high-temperature, high-ZT thermoelectric material. Joule 2, 698-709 (2018).

46. May, A. F., Flage-Larsen, E. \& Snyder, G. J. Electron and phonon scattering in the high-temperature thermoelectric $\mathrm{La}_{3} \mathrm{Te}_{4-z} \mathrm{M}_{\mathrm{z}}(\mathrm{M}=\mathrm{Sb}, \mathrm{Bi})$. Phys. Rev. B 81, 125205 (2010).

47. Toberer, E. S. et al. Traversing the metal-insulator transition in a Zintl phase: rational enhancement of thermoelectric efficiency in $\mathrm{Yb}_{14} \mathrm{Mn}_{1-x} \mathrm{Al}_{\mathrm{x}} \mathrm{Sb}_{11}$. Adv. Funct. Mater. 18, 2795-2800 (2008)

48. Xin, J. et al. Valleytronics in thermoelectric materials. npj Quant. Mater. 3, 9 (2018).

49. Fu, C. et al. Realizing high figure of merit in heavy-band p-type half-Heusler thermoelectric materials. Nat. Commun. 6, 8144 (2015).

50. Yan, X. et al. Thermoelectric property study of nanostructured p-type HalfHeuslers (Hf, Zr, Ti)CoSb ${ }_{0.8} \mathrm{Sn}_{0.2}$. Adv. Energy Mater. 3, 1195-1200 (2013).
51. Parker, W., Jenkins, R., Butler, C. \& Abbott, G. Flash method of determining thermal diffusivity, heat capacity, and thermal conductivity. J. Appl. Phys. 32, 1679-1684 (1961).

52. LaLonde, A. D., Pei, Y. \& Snyder, G. J. Reevaluation of $\mathrm{PbTe}_{1-x} \mathrm{I}_{x}$ as high performance n-type thermoelectric material. Energy Environ. Sci. 4, 2090-2096 (2011).

53. Tan, G. et al. Non-equilibrium processing leads to record high thermoelectric figure of merit in PbTe-SrTe. Nat. Commun. 7, 12167 (2016).

54. Zhang, J. et al. Extraordinary thermoelectric performance realized in n-type $\mathrm{PbTe}$ through multiphase nanostructure engineering. Adv. Mater. 29, 1703148 (2017).

55. Fu, L. et al. Large enhancement of thermoelectric properties in n-type $\mathrm{PbTe}$ via dual-site point defects. Energy Environ. Sci. 10, 2030-2040 (2017).

56. Bozin, E. S. et al. Entropically stabilized local dipole formation in lead chalcogenides. Science 330, 1660-1663 (2010).

57. Delaire, O. et al. Giant anharmonic phonon scattering in PbTe. Nat. Mater. 10, 614-619 (2011).

58. Chang, C. \& Zhao, L.-D. Anharmoncity and low thermal conductivity in thermoelectrics. Mater. Today Phys. 4, 50-57 (2018).

59. Biswas, K. et al. High-performance bulk thermoelectrics with all-scale hierarchical architectures. Nature 489, 414-418 (2012).

60. Zhao, L.-D. et al. All-scale hierarchical thermoelectrics: MgTe in PbTe facilitates valence band convergence and suppresses bipolar thermal transport for high performance. Energy Environ. Sci. 6, 3346-3355 (2013).

61. $\mathrm{Wu}, \mathrm{H}$. J. et al. Broad temperature plateau for thermoelectric figure of merit $\mathrm{ZT}>2$ in phase-separated $\mathrm{PbTe}_{0.7} \mathrm{~S}_{0.3}$. Nat. Commun. 5, 4515 (2014).

62. Pei, Y. et al. Convergence of electronic bands for high performance bulk thermoelectrics. Nature 473, 66-69 (2011).

63. $\mathrm{Wu}, \mathrm{D}$. et al. Superior thermoelectric performance in PbTe-PbS pseudo-binary: extremely low thermal conductivity and modulated carrier concentration. Energy Environ. Sci. 8, 2056-2068 (2015).

64. Heremans, J. P. et al. Enhancement of thermoelectric efficiency in PbTe by distortion of the electronic density of states. Science 321, 554-557 (2008).

65. Biswas, K. et al. Strained endotaxial nanostructures with high thermoelectric figure of merit. Nat. Chem. 3, 160-166 (2011).

66. Tan, $\mathrm{G}$. et al. Subtle roles of $\mathrm{Sb}$ and $\mathrm{S}$ in regulating the thermoelectric properties of n-type PbTe to high performance. Adv. Energy Mater. 7, 1700099 (2017).

67. Zhang, Q. et al. Deep defect level engineering: a strategy of optimizing the carrier concentration for high thermoelectric performance. Energy Environ. Sci. 11, 933-940 (2018).

68. Skipetrov, E., Zvereva, E., Volkova, O., Slyn'ko, E. \& Mousalitin, A. On Fermi level pinning in lead telluride based alloys doped with mixed valence impurities. Mater. Sci. Eng. B 91, 416-420 (2002).

69. Pei, Y., Wang, H. \& Snyder, G. J. Band engineering of thermoelectric materials. Adv. Mater. 24, 6125-6135 (2012).

70. Zhao, L.-D., Dravid, V. P. \& Kanatzidis, M. G. The panoscopic approach to high performance thermoelectrics. Energy Environ. Sci. 7, 251-268 (2014).

71. Crocker, A. \& Rogers, L. Valence band structure of PbTe. Le. J. De. Phys. Colloq. 29, 129-132 (1968).

72. Girard, S. N. et al. High performance Na-doped PbTe-PbS thermoelectric materials: electronic density of states modification and shape-controlled nanostructures. J. Am. Chem. Soc. 133, 16588-16597 (2011).

73. Heremans, J. P., Wiendlocha, B. \& Chamoire, A. M. Resonant levels in bulk thermoelectric semiconductors. Energy Environ. Sci. 5, 5510-5530 (2012).

74. Correa Baena, J. P. et al. Highly efficient planar perovskite solar cells through band alignment engineering. Energy Environ. Sci. 8, 2928-2934 (2015).

75. Chuang, C.-H. M., Brown, P. R., Bulović, V. \& Bawendi, M. G. Improved performance and stability in quantum dot solar cells through band alignment engineering. Nat. Mater. 13, 796-801 (2014).

76. Rowe, D. M. CRC Handbook of Thermoelectrics (CRC press, 1995).

77. Xiao, Y. et al. Realizing high performance $n$-type PbTe by synergistically optimizing effective mass and carrier mobility and suppressing bipolar thermal conductivity. Energy Environ. Sci. 11, 2486-2495 (2018).

78. Kane, E. O. Band structure of indium antimonide. J. Phys. Chem. Solids 1, 249-261 (1957).

79. Pei, Y., LaLonde, A. D., Wang, H. \& Snyder, G. J. Low effective mass leading to high thermoelectric performance. Energy Environ. Sci. 5, 7963-7969 (2012).

80. Volkov, B. A., Ryabova, L. I. \& Khokhlov, D. R. Mixed-valence impurities in lead telluride-based solid solutions. Phys. Usp. 45, 819-846 (2002).

81. Kaĭdanov, V. \& Ravich, Y. I. Deep and resonance states in $A^{I V} B^{\mathrm{VI}}$ semiconductors. Phys. Usp. 28, 31-53 (1985).

82. Su, X. et al. Weak electron phonon coupling and deep level impurity for high thermoelectric performance $\mathrm{Pb}_{1-x} \mathrm{Ga}_{x} T$ e. Adv. Energy Mater. 8, 1800659 (2018).

83. Nielsen, M., Levin, E., Jaworski, C., Schmidt-Rohr, K. \& Heremans, J. Chromium as resonant donor impurity in PbTe. Phys. Rev. B 85, 045210 (2012). 
84. Skipetrov, E., Kruleveckaya, O., Skipetrova, L., Slynko, E. \& Slynko, V. Fermi level pinning in Fe-doped PbTe under pressure. Appl. Phys. Lett. 105, 022101 (2014).

85. Wiendlocha, B. Localization and magnetism of the resonant impurity states in $\mathrm{Ti}$ doped PbTe. Appl. Phys. Lett. 105, 133901 (2014).

86. Skipetrov, E., Skipetrova, L., Knotko, A., Slynko, E. \& Slynko, V. Scandium resonant impurity level in PbTe. J. Appl. Phys. 115, 133702 (2014).

87. Harman, T. C., Taylor, P. J., Spears, D. L. \& Walsh, M. P. Thermoelectric quantumdot superlattices with high ZT. J. Electron. Mater. 29, L1-L2 (2000).

88. He, J., Girard, S. N., Kanatzidis, M. G. \& Dravid, V. P. Microstructure-lattice thermal conductivity correlation in nanostructured $\mathrm{PbTe}_{0.7} \mathrm{~S}_{0.3}$ thermoelectric materials. Adv. Funct. Mater. 20, 764-772 (2010).

89. Feng, D. et al. Investigation into the extremely low thermal conductivity in Ba heavily doped BiCuSeO. Nano Energy 27, 167-174 (2016).

90. Zhou, X. et al. Routes for high-performance thermoelectric materials. Mater. Today. https://doi.org/10.1016/j.mattod.2018.03.039 (2018).

91. Biswas, K. et al. High thermoelectric figure of merit in nanostructured p-type PbTe-MTe ( $\mathrm{M}=\mathrm{Ca}$, Ba). Energy Environ. Sci. 4, 4675-4684 (2011).

92. Pei, Y. Z., LaLonde, A. D., Heinz, N. A. \& Snyder, G. J. High thermoelectric figure of merit in PbTe alloys demonstrated in PbTe-CdTe. Adv. Energy Mater. 2, 670-675 (2012).

93. Bukala, M., Sankowski, P., Buczko, R. \& Kacman, P. Structural and electronic properties of $\mathrm{Pb}_{1-x} \mathrm{Cd}_{\mathrm{x}} \mathrm{Te}$ and $\mathrm{Pb}_{1-x} \mathrm{Mn}_{\mathrm{x}}$ Te ternary alloys. Phys. Rev. B 86, 085205 (2012).

94. Shi, X. et al. Solid solubility of Ir and Rh at the Co sites of skutterudites. J. Appl. Phys. 101, 123525 (2007).

95. Ginting, D. et al. Enhancement of thermoelectric performance in Na-doped $\mathrm{Pb}_{0.6} \mathrm{Sn}_{0.4} \mathrm{Te}_{0.95-x} \mathrm{Se}_{x} \mathrm{~S}_{0.05}$ via breaking the inversion symmetry, band convergence, and nanostructuring by multiple elements doping. ACS Appl. Mater. Interfaces 10, 11613-11622 (2018).

96. He, J. et al. Morphology control of nanostructures: Na-Doped PbTe-PbS system. Nano Lett. 12, 5979-5984 (2012).

97. Zhou, M., Li, J.-F. \& Kita, T. Nanostructured $\mathrm{AgPb}_{\mathrm{m}} \mathrm{SbTe}_{\mathrm{m}+2}$ system bulk materials with enhanced thermoelectric performance. J. Am. Chem. Soc. 130, 4527-4532 (2008)

98. Xiao, Y. et al. Remarkable roles of $\mathrm{Cu}$ to synergistically optimize phonon and carrier transport in n-type PbTe-Cu 2 Te. J. Am. Chem. Soc. 139, 18732-18738 (2017).

99. Tan, G. et al. High thermoelectric performance of p-type SnTe via a synergistic band engineering and nanostructuring approach. J. Am. Chem. Soc. 136, 7006-7017 (2014).

100. Liu, Y. et al. Large enhancements of thermopower and carrier mobility in quantum dot engineered bulk semiconductors. J. Am. Chem. Soc. 135, 7486-7495 (2013).

101. Kudman, I. Thermoelectric properties of dilute PbTe-GeTe alloys. Metall. Trans. 2, 163-168 (1971).

102. Guéguen, $\mathrm{A}$. et al. Thermoelectric properties and nanostructuring in the p-type materials $\mathrm{NaPb}_{18-x} \mathrm{Sn}_{x} \mathrm{MTe}_{20}(\mathrm{M}=\mathrm{Sb}, \mathrm{Bi})$. Chem. Mater. 21, 1683-1694 (2009).

103. Androulakis, J. et al. Nanostructuring and high thermoelectric efficiency in ptype $\mathrm{Ag}\left(\mathrm{Pb}_{1-\mathrm{y}} \mathrm{Sn}_{\mathrm{y}}\right)_{\mathrm{m}} \mathrm{SbTe}_{2+\mathrm{m}}$. Adv. Mater. 18, 1170-1173 (2006).

104. Zhang, Y., Wu, L., Zhang, J., Xing, J. \& Luo, J. Eutectic microstructures and thermoelectric properties of MnTe-rich precipitates hardened PbTe. Acta Mater. 111, 202-209 (2016)

105. Zhang, Q. et al. Heavy doping and band engineering by potassium to improve the thermoelectric figure of merit in p-type $\mathrm{PbTe}, \mathrm{PbSe}$, and $\mathrm{PbTe}_{1-\mathrm{y}} \mathrm{Se}_{\mathrm{y}} . \mathrm{J}$. Am. Chem. Soc. 134, 10031-10038 (2012).

106. Wang, $\mathrm{H}$. et al. High thermoelectric performance of a heterogeneous $\mathrm{PbTe}$ nanocomposite. Chem. Mater. 27, 944-949 (2015).
107. Wang, $\mathrm{H}$. et al. Right sizes of nano- and microstructures for high-performance and rigid bulk thermoelectrics. Proc. Natl Acad. Sci. USA 111, 10949-10954 (2014).

108. Gelbstein, Y. \& Davidow, J. Highly efficient functional $\mathrm{Ge}_{x} \mathrm{~Pb}_{1-\mathrm{x}} \mathrm{Te}$ based thermoelectric alloys. Phys. Chem. Chem. Phys. 16, 20120-20126 (2014).

109. Korkosz, R. J. et al. High ZT in p-type $(\mathrm{PbTe})_{1-2 x}(\mathrm{PbSe})_{x}(\mathrm{PbS})_{x}$ thermoelectric materials. J. Am. Chem. Soc. 136, 3225-3237 (2014).

110. Ahn, K. et al. Enhanced thermoelectric properties of p-type nanostructured PbTe-MTe ( $\mathrm{M}=\mathrm{Cd}, \mathrm{Hg})$ materials. Energy Environ. Sci. 6, 1529-1537 (2013).

111. Jian, Z. Z. et al. Significant band engineering effect of YbTe for high performance thermoelectric PbTe. J. Mater. Chem. C. 3, 12410-12417 (2015).

112. Kim, Y.-J., Zhao, L.-D., Kanatzidis, M. G. \& Seidman, D. N. Analysis of nanoprecipitates in a Na-doped PbTe-SrTe thermoelectric material with a high figure of merit. ACS Appl. Mater. Interfaces 9, 21791-21797 (2017).

113. Chen, $\mathrm{Z}$. et al. Lattice dislocations enhancing thermoelectric $\mathrm{PbTe}$ in addition to band convergence. Adv. Mater. 29, 1606768 (2017).

114. Chere, E. K. et al. Enhancement of thermoelectric performance in n-type $\mathrm{PbTe}_{1}$ ${ }_{-y} \mathrm{Se}_{y}$ by doping $\mathrm{Cr}$ and tuning Te:Se ratio. Nano Energy 13, 355-367 (2015).

115. Girard, S. N. et al. PbTe-PbSnS, thermoelectric composites: low lattice thermal conductivity from large microstructures. Energy Environ. Sci. 5, 8716-8725 (2012).

116. Cohen, I., Kaller, M., Komisarchik, G., Fuks, D. \& Gelbstein, Y. Enhancement of the thermoelectric properties of $\mathrm{n}$-type $\mathrm{PbTe}$ by $\mathrm{Na}$ and $\mathrm{Cl}$ co-doping. J. Mater. Chem. C. 3, 9559-9564 (2015).

117. Rawat, P. K., Paul, B. \& Banerji, P. Exploration of $\mathrm{Zn}$ resonance levels and thermoelectric properties in I-doped PbTe with ZnTe nanostructures. ACS Appl. Mater. Interfaces 6, 3995-4004 (2014).

118. Ahn, K. et al. Exploring resonance levels and nanostructuring in the $\mathrm{PbTe}-\mathrm{CdTe}$ system and enhancement of the thermoelectric figure of merit. J. Am. Chem. Soc. 132, 5227-5235 (2010).

119. Xiao, Y. et al. Synergistically optimizing thermoelectric transport properties of $n$ type PbTe via Se and Sn co-alloying. J. Alloy. Compd. 724, 208-221 (2017).

120. Jood, P. et al. Enhanced average thermoelectric figure of merit of $\mathrm{n}$-type $\mathrm{PbTe}_{1}$

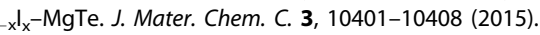

121. Ginting, D. et al. High thermoelectric performance due to nano-inclusions and randomly distributed interface potentials in N-type ( $\mathrm{PbTe}_{0.93}$ $\left.{ }_{x} \mathrm{Se}_{0.07} \mathrm{Cl}_{x}\right)_{0.93}(\mathrm{PbS})_{0.07}$ composites. J. Mater. Chem. A 5, 13535-13543 (2017).

122. Ding, G., Si, J., Yang, S., Wang, G. \& Wu, H. High thermoelectric properties of $n-$ type Cd-doped PbTe prepared by melt spinning. Scr. Mater. 122, 1-4 (2016).

123. Pei, Y. et al. Optimum carrier concentration in n-type PbTe thermoelectrics. Adv. Energy Mater. 4, 1400486 (2014).

(c) Open Access This article is licensed under a Creative Commons Attribution 4.0 International License, which permits use, sharing, adaptation, distribution and reproduction in any medium or format, as long as you give appropriate credit to the original author(s) and the source, provide a link to the Creative Commons license, and indicate if changes were made. The images or other third party material in this article are included in the article's Creative Commons license, unless indicated otherwise in a credit line to the material. If material is not included in the article's Creative Commons license and your intended use is not permitted by statutory regulation or exceeds the permitted use, you will need to obtain permission directly from the copyright holder. To view a copy of this license, visit http://creativecommons. org/licenses/by/4.0/.

(c) The Author(s) 2018 